\title{
A Proximal Interior Point Algorithm with Applications to Image Processing
}

\author{
Emilie Chouzenoux - Marie-Caroline Corbineau - Jean-Christophe \\ Pesquet
}

Received: date / Accepted: date

\begin{abstract}
In this article, we introduce a new proximal interior point algorithm (PIPA). This algorithm is able to handle convex optimization problems involving various constraints where the objective function is the sum of a Lipschitz differentiable term and a possibly nonsmooth one. Each iteration of PIPA involves the minimization of a merit function evaluated for decaying values of a logarithmic barrier parameter. This inner minimization is performed thanks to a finite number of subiterations of a variable metric forward-backward method employing a line search strategy. The convergence of this latter step as well as the convergence the global method itself are analyzed. The numerical efficiency of the proposed approach is demonstrated in two image processing applications.
\end{abstract}

Keywords Interior point methods, proximity operator, constrained optimization, forward-backward algorithm, variable metric, line search, Armijo strategy, hyperspectral unmixing, geometry-texture decomposition

Emilie Chouzenoux ${ }^{1}$

E-mail: emilie.chouzenoux@centralesupelec.fr

Marie-Caroline Corbineau ${ }^{1}$ (corresponding author)

E-mail: mariecaroline_corbineau@hotmail.fr

Jean-Christophe Pesquet ${ }^{1}$

E-mail: jean-christophe.pesquet@centralesupelec.fr

${ }^{1}$ CVN, CentraleSupélec, Inria Saclay, University ParisSaclay, 9 rue Joliot Curie, Gif-sur-Yvette, 91190, France

\section{Introduction}

Many problems in image processing, such as segmentation [1], classification [2], or restoration [3], can be formulated as the minimization of a convex objective function under convex constraints. This type of problem can be successfully addressed by interior point methods (IPMs) $[4,5,6]$. Instead of tackling directly the original optimization problem, IPMs solve a sequence of intermediate problems parametrized by a barrier parameter going to zero. In each intermediate problem, the original objective function is penalized with a logarithmic barrier, which is unbounded at the boundary of the feasible set. This modified objective function is referred to as a merit function. Thanks to the introduction of the barrier term, IPMs produce only strictly feasible iterates, which can be beneficial from the application viewpoint and also boost convergence. For instance, under suitable assumptions, the primal-dual interior point algorithm proposed in [7] presents superlinear convergence in the context of nonlinear programming. From a numerical perspective, IPMs have demonstrated very good performance on several challenging applications, such as image reconstruction [8] and multispectral image unmixing [9]. It is worth noting that most of interior point approaches rely on first or second-order methods and, therefore, assume that the objective function is at least twice-differentiable [10, 11].

However, in many image processing applications, the quality of the solution and its robustness to noise may be improved by including a nondifferentiable regularization term in the objective function. For instance, the $\ell_{1}$-norm promotes sparsity [12], while the total variation semi-norm leads to almost piecewise constant solutions, which is at the core of variational segmentation and decomposition models [13]. Although IPMs can handle 
the $\ell_{1}$ norm $[14,15,16]$, for more general non-smooth penalizations, approaches relying on the proximity operator $^{1}$ seem more appropriate [17].

In most applications, the objective function is actually composite, in that it can be split into a nonsmooth term and a differentiable term. Some proximal resolution methods take advantage of this decomposition [17], such as the widely-used forward-backward (FB) algorithm [18], where each iteration is made of two steps, namely a gradient step (i.e., forward) on the differentiable term, and a proximal step (i.e. backward) on the nonsmooth term. The FB algorithm can be accelerated by using a so-called variable metric or preconditioner. For instance, in [19, 20], the convergence of a variable metric forward-backward (VMFB) method is established in the convex setting under a monotonicity condition. Recent works [21, 22] relying on the Kurdyka-Łojasiewicz (KL) inequality [23, 24, 25] extend this convergence result to nonconvex problems, under milder boundedness condition on the variable metrics. The KL property is also useful for deriving convergence rates [26, 27].

Proximal and interior point methods can be combined in efficient solvers, as illustrated in [28], where the authors proposed an algorithm that minimizes a convex differentiable function over convex inequality constraints. However, this framework does not make use of any splitting strategy, hence it assumes that the proximity operator of the merit function is easy to compute. More recently, a preconditioning strategy based on the logarithmic barrier was proposed in [29] to modify the dual update in a proximal primal-dual algorithm.

A major challenge, when dealing with IPMs is that the logarithmic barrier does not satisfy the gradientLipschitz property. Therefore, specific line search strategies have to be designed in order to preserve the convergence properties of the methods used to solve the subproblems [30]. This question has also been addressed in the context of VMFB algorithms, when the gradient of the smooth term in the objective function is not globally Lipschitz-continuous. Following the work of [31], Armijo-type line searches were proposed in [32, 33], where the convergence of the algorithm is obtained in a convex setting under suitable assumptions on the variable metrics. However, the line search strategy in [32] requires multiple gradient computations, while [33] requires the domain of the nondifferentiable function to be closed. Other line searches have also been studied in [34], where convergence guarantees and convergence rate in terms of function values are provided for the convex case. It is worth noting that, similarly to [35], [34] considers a relaxed version of the monotonicity condi-

\footnotetext{
1 see http://proximity-operator.net/
}

tion on the variable metrics from [20], where the metrics converge to a multiple of the identity operator, with a multiplicative factor which is allowed to vary along iterations. This assumption still remains restrictive compared to the conditions required in [21], where the proof of convergence is carried out using the KL property. In this paper, we propose to combine the VMFB algorithm with the logarithmic barrier method, leading to a proximal interior point algorithm, which is referred to as PIPA. We will make use of one of the line searches investigated in [34] to determine the stepsize value for each iteration of the proposed algorithm. Since our assumptions on the function domains are different from those in [34], we prove that the line search remains valid in our context. Furthermore, we carry out the convergence analysis of the proposed algorithm under a mild boundedness condition on the involved variable metrics. Under some additional assumptions, we derive a linear convergence rate for the inner loop involved in PIPA. We show that the proposed algorithm performs well with respect to state-of-the-art methods on two applications in image processing, namely hyperspectral unmixing and joint geometry-texture decomposition and reconstruction of computed tomography (CT) data. Our numerical experiments demonstrate in addition the benefits of using a variable metric to accelerate the convergence of PIPA.

The article is organized as follows. Mathematical notation and definitions are provided in Section 2, where the proposed method is detailed and summarized in Algorithms 1 and 2. Section 3 is dedicated to the mathematical analysis of PIPA. Our main theoretical results are provided in Section 3.1, while the proofs for these results are given in the following sections. In Section 3.2 we show that the line search and inner loop in PIPA are well-defined, and in Section 3.3 we demonstrate the linear convergence rate of Algorithm 1 for a useful case where the KL property is satisfied. In Section 3.4 we prove the convergence results regarding PIPA, i.e. Algorithm 2. Numerical experiments are presented in Section 4 and some conclusions are drawn in Section 5 .

\section{Proximal interior point method}

\subsection{Notation and definitions}

In this paper, $\mathbb{R}^{n}$ denotes the $n$-dimensional Euclidean space endowed with the standard scalar product $\langle\cdot, \cdot\rangle$ and the norm $\|\cdot\|$. The set of symmetric positive definite matrices in $\mathbb{R}^{n \times n}$ is referred to as $\mathfrak{S}_{n}$. For every matrix $A \in \mathfrak{S}_{n},\|A\|$ denotes its spectral norm and $\|\cdot\|_{A}$ denotes the norm induced by $A$, i.e. $\|\cdot\|_{A}=\langle\cdot, A \cdot\rangle^{1 / 2}$. In addition, $\preceq$ refers to the Loewner partial order, i.e. for 
every $A$ and $B$ in $\mathfrak{S}_{n}, A \preceq B$ if and only if $B-A$ is positive semidefinite. The identity matrix in $\mathbb{R}^{n \times n}$ is denoted as $\mathbb{\square}_{n}$. For every function $f: \mathbb{R}^{n} \rightarrow \mathbb{R} \cup\{+\infty\}$, its domain is defined as $\operatorname{dom} f=\left\{x \in \mathbb{R}^{n} \mid f(x) \neq+\infty\right\}$. A function is said proper if its domain is nonempty. The set of functions which are proper, convex, lower semicontinuous (lsc) on $\mathbb{R}^{n}$ and take values in $\mathbb{R} \cup\{+\infty\}$, is denoted as $\Gamma_{0}\left(\mathbb{R}^{n}\right)$. For every set $\mathcal{D} \subset \mathbb{R}^{n}, \overline{\mathcal{D}}$ denotes its closure.

Definition 1 (Lipschitz-continuity) A function $f$ : $\mathbb{R}^{n} \rightarrow \mathbb{R}^{p}$ is said Lipschitz-continuous if there exists $L \geq 0$ such that

$\left(\forall(x, y) \in(\operatorname{dom} f)^{2}\right) \quad\|f(x)-f(y)\| \leq L\|x-y\|$.

The proximity operator [36, 37], which is defined below, is a fundamental tool in convex analysis [17, 38, 39].

Definition 2 (Proximity operator) For every $f \in$ $\Gamma_{0}\left(\mathbb{R}^{n}\right), A \in \mathfrak{S}_{n}$ and $x \in \mathbb{R}^{n}$, the proximity operator of $f$ at $x$, with regards to the norm induced by $A$, is defined as

$\operatorname{prox}_{f}^{A}(x)=\operatorname{argmin}_{y \in \mathbb{R}^{n}}\left(f(y)+\frac{1}{2}\|x-y\|_{A}^{2}\right)$.

In Definition 2, when $A$ is not specified, then the standard Euclidean norm is used, i.e. $A=\mathbb{\unlhd}_{n}$. If $\operatorname{prox}_{f}$ is simple to compute, then the solution to (1) for an arbitrary $A \in \mathfrak{S}_{n}$ can be obtained by using the dual forward-backward (DFB) algorithm [40] or its accelerated version [41].

Definition 3 (Moreau Subdifferential) Let $f$ : $\mathbb{R}^{n} \rightarrow \mathbb{R} \cup\{+\infty\}$ be proper. The subdifferential of $f$ is the set-valued operator $\partial f$ such that, for every $x \in \mathbb{R}^{n}$,

$\partial f(x)=\left\{u \in \mathbb{R}^{n} \mid\left(\forall y \in \mathbb{R}^{n}\right)\langle y-x, u\rangle+f(x) \leq f(y)\right\}$.

The subdifferential, defined hereabove, provides an equivalent characterization for the proximity operator. From [37, Proposition 16.44], it follows that for every $f \in$ $\Gamma_{0}\left(\mathbb{R}^{n}\right), A \in \mathfrak{S}_{n}, x \in \mathbb{R}^{n}$, and $z \in \mathbb{R}^{n}$

$z=\operatorname{prox}_{f}^{A}(x) \Longleftrightarrow x-z \in A^{-1} \partial f(z)$.

The proximity operator exhibits many useful properties, in particular it is firmly nonexpansive [37, Proposition 12.28]. Moreover, by combining Fermat's rule [37, Theorem 16.3] with (2), for every functions $f$ and $g$ in $\Gamma_{0}\left(\mathbb{R}^{n}\right)$, if $g$ is differentiable on a neighborhood of $x \in \mathbb{R}^{n}$, then $x$ is a minimizer of $f+g$ if and only if $x=\operatorname{prox}_{f}(x-\nabla g(x))$.
Definition 4 For every $\eta>0, \Phi_{\eta}$ is the set of continuous concave functions $\phi:[0, \eta[\rightarrow[0,+\infty[$ such that $\phi(0)=0, \phi$ is $\mathcal{C}^{1}$ on $] 0, \eta[$ and continuous at 0 and, for every $s \in] 0, \eta\left[, \phi^{\prime}(s)>0\right.$.

From the above definition, we can state the so-called Kurdyka-Lojasiewicz (KL) property. Following the seminal work of Łojasiewicz and Kurdyka, the KL property has been extensively used for proving the convergence and obtaining convergence rates of optimization methods for possibly nondifferentiable functions, both in the convex and nonconvex case [25, 42].

Definition 5 (KL property [42]) Let $f: \mathbb{R}^{n} \rightarrow$ $\mathbb{R} \cup\{+\infty\}$ be proper and lower semicontinuous. The function $f$ is said to have the Kurdyka-Lojasiewicz (KL) property at $\bar{x} \in \operatorname{dom} \partial f=\left\{x \in \mathbb{R}^{n} \mid \partial f(x) \neq \varnothing\right\}$ if there exist $\eta \in] 0,+\infty]$, a neighborhood $\Omega$ of $\bar{x}$ and a function $\phi \in \Phi_{\eta}$, such that for all $x \in \Omega$ such that $f(\bar{x})<f(x)<f(\bar{x})+\eta$, the following inequality holds

$\phi^{\prime}(f(x)-f(\bar{x})) \operatorname{dist}(0, \partial f(x)) \geq 1$.

If $f$ satisfies the KL property at each point of $\operatorname{dom} \partial f$, then $f$ is called a $K L$ function.

The KL property is satisfied in most practical optimization applications and, for a wide class of functions, the reparametrization $\phi$ has a specific form and can be written $\left(\forall s \in\left[0, \eta[) \phi(s)=\bar{c} s^{1-\alpha}\right.\right.$ for some $\bar{c}>0$ and $\alpha \in[0,1[$. This parameter $\alpha$ is called the $K L$ exponent and can be made explicit under some conditions, as detailed in [43]. This is of particular interest because this exponent can help derive convergence rates for many optimization methods, as proven for instance in [26, Theorem 2] or [44, Theorem 3.4].

\subsection{Optimization problem and assumptions}

In this paper we consider the following constrained convex minimization problem,

$$
\begin{aligned}
\mathcal{P}_{0}: & \underset{x \in \mathbb{R}^{n}}{\operatorname{minimize}} f(x)+g(x) \\
& \text { subject to }(\forall i \in\{1, \ldots, p\}) \quad c_{i}(x) \leq 0,
\end{aligned}
$$

where $p>0$, and Assumption 1 below is satisfied.

\section{Assumption 1}

(i) The set of solutions to $\mathcal{P}_{0}$ is nonempty and bounded.

(ii) Functions $f, g$ and $\left(c_{i}\right)_{1 \leq i \leq p}$ belong to $\Gamma_{0}\left(\mathbb{R}^{n}\right)$, and $f+g$ is bounded from below. The set $\mathcal{D}=\left\{x \in \mathbb{R}^{n} \mid(\forall i \in\{1, \ldots, p\}) c_{i}(x)<0\right\}$ is assumed to be nonempty, open, and $\overline{\mathcal{D}} \subset \operatorname{dom} f \subset$ domg. 
(iii) Functions $g$ and $\left(c_{i}\right)_{1 \leq i \leq p}$ are differentiable on $\overline{\mathcal{D}}$; $\nabla g$ and $\left(\nabla c_{i}\right)_{1 \leq i \leq p}$ are Lipschitz-continuous on any compact subset of $\overline{\mathcal{D}}$.

Since the functions $\left(c_{i}\right)_{1 \leq i \leq p}$ are lsc, the closure of $\mathcal{D}$ is equal to $\overline{\mathcal{D}}=\left\{x \in \mathbb{R}^{n} \mid(\forall i \in\{1, \ldots, p\}) c_{i}(x) \leq 0\right\}$. In image processing, the constraints can be derived from the underlying geometry of the problem [45]. For instance, inequality constraints are used in a problem of deformable image matching in [46] to ensure that the estimated image deformation is injective and preserves the topology. Constraints can also serve to enforce some a priori knowledge about the solution, and act as regularization terms, as in the image segmentation approach in [47], where bound constraints are imposed on the segmented areas and their barycenters.

Following the framework of interior point methods, we propose to reformulate the constrained problem $\mathcal{P}_{0}$ as a sequence $\left(\mathcal{P}_{\mu_{j}}\right)_{j \in \mathbb{N}}$ of modified subproblems parametrized for every $j \in \mathbb{N}$ by a so-called barrier coefficient $\mu_{j}>0$ and defined as

$\mathcal{P}_{\mu_{j}}: \quad \underset{x \in \mathbb{R}^{n}}{\operatorname{minimize}} f(x)+g(x)+\mu_{j} \mathcal{B}(x)$

where $\mathcal{B}$ is the logarithmic barrier associated with the constraints:

$$
\begin{aligned}
\mathcal{B}: \mathbb{R}^{n} & \rightarrow \mathbb{R} \cup\{+\infty\} \\
x & \mapsto \begin{cases}-\sum_{i=1}^{p} \ln \left(-c_{i}(x)\right) & \text { if } x \in \mathcal{D} \\
+\infty & \text { otherwise. }\end{cases}
\end{aligned}
$$

For simplicity we introduce the shorter notation: $(\forall x \in$ $\left.\mathbb{R}^{n}\right) c(x)=\left(c_{i}(x)\right)_{1 \leq i \leq p} \in \mathbb{R}^{p}$, and

$(\forall \mu>0) \quad \varphi_{\mu}=g+\mu \mathcal{B}, \quad \Psi_{\mu}=f+\varphi_{\mu}$.

$\Psi_{\mu}$ is designated as merit function.

\subsection{PIPA algorithm}

The proposed method, PIPA, is made of two interlocked loops, which are detailed in Algorithms 1 and 2. Given $j \in \mathbb{N}$, Algorithm 1 produces an approximate solution to $\mathcal{P}_{\mu_{j}}$ via VMFB steps [21] consisting in a gradient step on the smooth term $\varphi_{\mu_{j}}$ and a proximal step on the nondifferentiable term $f$. The proximity operators are computed within the metric induced by symmetric definite positive preconditioning matrices whose eigenvalues are bounded from below and from above (using $\underline{\nu}$ and $\bar{\nu}$ positive constants). For well-chosen matrices, this so-called variable metric strategy can significantly improve the convergence speed. Preconditioning matrices
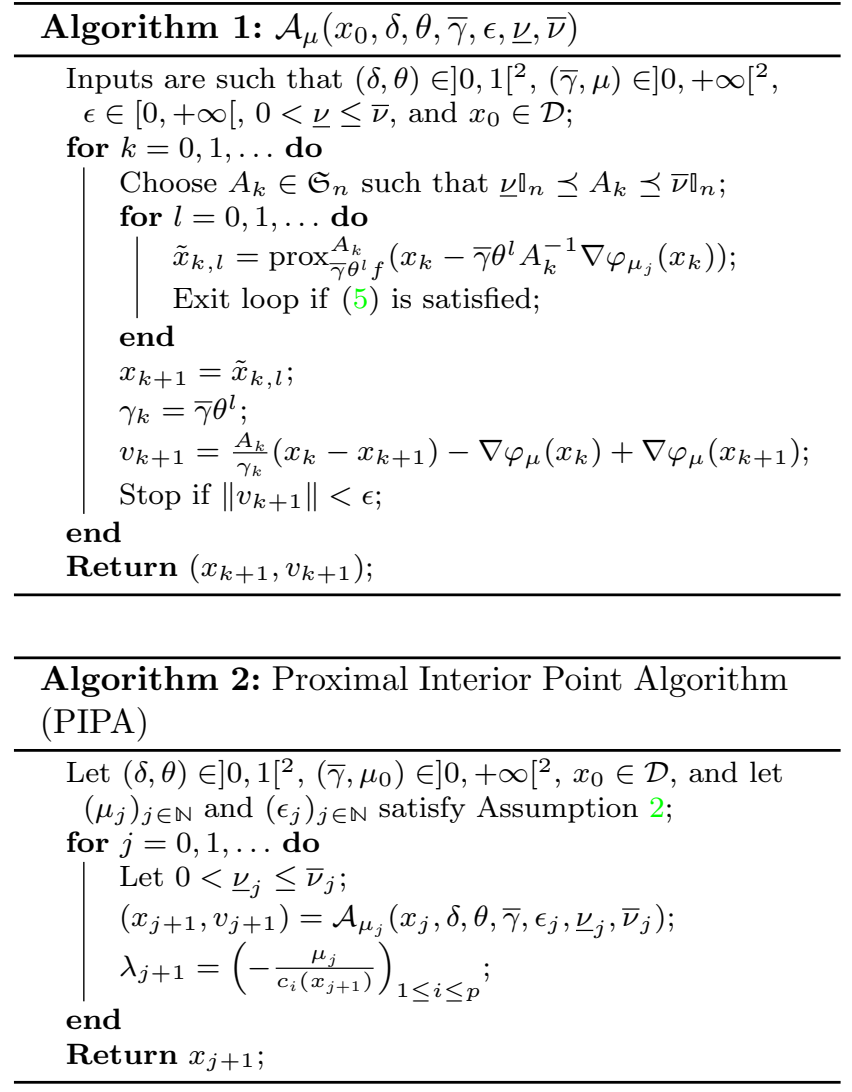

can be determined for instance through a majorizationminimization procedure [48]. It must be emphasized that, since the barrier is logarithmic, the gradient of $\varphi_{\mu_{j}}$ is not Lipschitz-continuous on $\mathbb{R}^{n}$. Thus, the VMFB algorithm must be associated with a line search to find an appropriate value for the stepsize so as to guarantee convergence of the scheme. In Algorithm 1, we use the backtracking line search method investigated in [34, LS1], itself a generalization of [32]. At iteration $k \in \mathbb{N}$, this backtracking procedure stops if

$$
\begin{gathered}
\varphi_{\mu}\left(\tilde{x}_{k, l}\right)-\varphi_{\mu}\left(x_{k}\right)-\left\langle\tilde{x}_{k, l}-x_{k}, \nabla \varphi_{\mu}\left(x_{k}\right)\right\rangle \leq \\
\frac{\delta}{\bar{\gamma} \theta^{l}}\left\|\tilde{x}_{k, l}-x_{k}\right\|_{A_{k}}^{2},
\end{gathered}
$$

for some $l \in \mathbb{N}$. Such line search ensures both sufficient decrease of the criterion and feasibility of the next iterate. It is interesting to note that if the whole cost function in $\mathcal{P}_{0}$ is smooth $(f=0)$, then it reduces to the standard Armijo line search along the steepest direction. Applications related to $\ell_{p}$-norms, with $1<p<2$, and Bregman distances where considered in [34], but, to the best of our knowledge, it is the first time that this line search is applied in the context of an interior point approach.

The resolution of the inner subproblem through Algorithm 1 is stopped once a certain accuracy is reached. 
In practice, we propose to stop the iterations once the norm of one element of the sequence $\left(v_{k+1}\right)_{k \in \mathbb{N}}$, where $(\forall k \in \mathbb{N}) v_{k+1} \in \partial \Psi_{\mu}\left(x_{k+1}\right)$, is sufficiently small. As stated in Assumption 2 below, the sequence $\left(\epsilon_{j}\right)_{j \in \mathbb{N}}$ and the barrier parameter $\left(\mu_{j}\right)_{j \in \mathbb{N}}$, have to be chosen properly to secure the convergence of the sequence of iterates produced by Algorithm 2 to a solution to the initial problem $\mathcal{P}_{0}$.

Assumption 2 (Hyperparameters) For every $j \in$ $\mathbb{N}, \mu_{j}>0$ and $\epsilon_{j}>0$. In addition, $\lim _{j \rightarrow+\infty} \mu_{j}=0$ and $\lim _{j \rightarrow+\infty} \epsilon_{j} / \mu_{j}=0$.

Remark 1 The sequence $\left(\lambda_{j+1}\right)_{j \in \mathbb{N}}$, produced by Algorithm 2 , is related to the Lagrangian associated with the constrained problem $\mathcal{P}_{0}$ and it is used in the proof of convergence as shown in Section 3.4.

\subsection{Related works}

It can be noted that there exist links between the proposed PIPA algorithm and a different class of methods called diagonal or penalization methods $[49,50]$, for which a general study was recently provided for the continuous setting in [51]. In [52], the authors proposed a similar approach in the discrete setting based on the FB algorithm. However, they assume that the gradient of the penalization function, which is the equivalent of our barrier, is Lipschitz continuous, and they let the penalization parameter tend to infinity, whereas our barrier parameter decreases to zero. In addition, their method does not solve a problem of the form (3), instead, it performs a hierarchical minimization [52, Eq. (6)]. This key difference is also highlighted in [53] and [54], in the continuous and discrete settings, respectively. In [54] the author studied the proximal point algorithm, without any forward-backward step, and considered a penalization parameter that, similarly to our barrier parameter, vanishes to zero. The optimization problem that is solved by this algorithm then depends on the rate of reduction chosen for this coefficient. In addition, it is assumed that the penalization function is bounded from below, which is not necessarily satisfied by the logarithmic barrier.

Related works also include Bregman distance approaches and entropy-like proximal algorithms [55, 56, 57], where the Euclidean norm in the definition of the proximity operator is replaced by a divergence measure. The latter can be chosen such that feasibility is ensured at each iteration. However, the computation of the modified proximity operator in such methods is usually not straightforward.

\section{Convergence analysis}

This section aims at studying the convergence of the proposed PIPA method. After stating our main results in Section 3.1, we provide the corresponding proofs in Sections 3.2, 3.3 and 3.4.

\subsection{Main results}

Theorem 1 guarantees that the stopping criterion in Algorithm 1 is well-defined.

Theorem 1 Under Assumption 1, for every $(\delta, \theta) \in$ ] $\left.0,1\left[{ }^{2},(\bar{\gamma}, \mu, \bar{\nu}) \in\right] 0,+\infty\left[{ }^{3}, \underline{\nu} \in\right] 0, \bar{\nu}\right]$, and $x_{0} \in \mathcal{D}$, for every $\epsilon>0$, there exists $k \in \mathbb{N}$ such that $\left\|v_{k+1}\right\|<\epsilon$.

Thus, an important feature of Algorithm 1 is the decay rate of the sequence $\left(\left\|v_{k+1}\right\|\right)_{k \in \mathbb{N}}$. This rate can be made explicit for the particular instance of linear inequality constraints. More precisely we focus on the case when the constrained problem takes the form:

$$
\begin{aligned}
& \underset{x \in \mathbb{R}^{n}}{\operatorname{minimize}} \widetilde{g}(H x)+f(x) \\
& \text { subject to } M x+m \in]-\infty, 0]^{p},
\end{aligned}
$$

where $\left.\left.\widetilde{g}: \mathbb{R}^{s} \rightarrow\right]-\infty,+\infty\right], H \in \mathbb{R}^{s \times n}, M \in \mathbb{R}^{p \times n}, m \in$ $\mathbb{R}^{p}$, and the involved functionals satisfy the following assumption.

\section{Assumption 3}

(i) $f$ is a polyhedral function, i.e. its epigraph a finite intersection of closed halfspaces.

(ii) $\widetilde{g}$ is lsc with an open domain, it is strongly convex on any compact subset of $\operatorname{dom} \widetilde{g}$ and it is twice continuously differentiable on $\operatorname{dom} \tilde{g}$.

(iii) $f+\widetilde{g} \circ H$ is proper and it is continuous on the domain of its subdifferential.

Remark 2 Assumption 3 holds for instance if $\widetilde{g}=\frac{1}{2}\|\cdot-y\|^{2}$ with $y \in \mathbb{R}^{s}$ and if $f=\kappa\|W \cdot\|_{1}$ where $\kappa \geq 0$ and $W \in \mathbb{R}^{q \times n}$ is a linear transform (e.g., a wavelet analysis operator $[58,59])$. This corresponds to an $\ell_{1}$-regularized least-squares problem, at the core of many applications such as denoising [60], image restoration [14], machine learning, or biological data analysis [61, Chapter 18.4].

The following result can be deduced from existing results concerning the use of the KL inequality in optimization.

Theorem 2 Let $\mu>0$ and consider the barrier problem $\mathcal{P}_{\mu}$ associated to problem (6). Under Assumptions 1 and 3, for every $(\delta, \theta) \in] 0,1\left[^{2},(\bar{\gamma}, \bar{\nu}) \in\right] 0,+\infty\left[^{2}, \underline{\nu} \in\right.$ ] $0, \bar{\nu}]$, and $x_{0} \in \mathcal{D}$, the sequence $\left(v_{k+1}\right)_{k \in \mathbb{N}}$ generated by Algorithm 1 converges linearly to 0 when $\epsilon=0$. 
We finally present results regarding the convergence of the proposed method PIPA, i.e. Algorithm 2.

Theorem 3 Suppose that Assumptions 1 and 2 hold for every $(\delta, \theta) \in] 0,1\left[{ }^{2},\left(\bar{\gamma}, \mu_{0}\right) \in\right] 0,+\infty\left[^{2}\right.$ and $x_{0} \in \mathcal{D}$. Then, any sequence $\left(x_{j+1}, \lambda_{j+1}\right)_{j \in \mathbb{N}}$ generated by Algorithm 2 is bounded. In addition, every of its cluster point $\left(x^{*}, \lambda^{*}\right)$ is a primal-dual solution to $\mathcal{P}_{0}$, i.e. $\left(x^{*}, \lambda^{*}\right)$ is a saddle point for the Lagrangian defined in $(30)$.

A stronger convergence result can be obtained, under additional assumptions. In particular the following one will turn out to play an important role.

Assumption 4 Either the constraints are affine, i.e. $c: x \mapsto M x+m$ where $M \in \mathbb{R}^{p \times n}$ and $m \in \mathbb{R}^{p}$, and $M$ has full column rank (i.e. $M$ is injective), or there exists $i \in\{1, \ldots, p\}$ such that $c_{i}$ is strictly convex.

Let $\mathcal{S}_{P}$ be the set of solutions to $\mathcal{P}_{0}$ (primal solutions), and let $\mathcal{S}_{D}$ be the set of solutions to the Lagrange dual problem associated with $\mathcal{P}_{0}$ (dual solutions) [62, Section 5.2]. In addition, let

$J_{P}=\left\{i \in\{1, \ldots, p\} \mid\left(\exists x \in \mathcal{S}_{P}\right) c_{i}(x)<0\right\}$,

$J_{D}=\left\{i \in\{1, \ldots, p\} \mid\left(\exists \lambda=\left(\lambda^{(\ell)}\right)_{1 \leq \ell \leq p} \in \mathcal{S}_{D}\right) \lambda^{(i)}>0\right\}$

Our main convergence result, summarized in Theorem 4, provides a useful characterization for the limit point of Algorithm 2 using the so-called analytic center, which will be introduced in Section 3.4.2.

Theorem 4 Under Assumptions 1 and 2 the following hold.

(i) If there exists only one element in $\mathcal{S}_{P}$ (resp. $\left.\mathcal{S}_{D}\right)$ then the sequence $\left(x_{j+1}\right)_{j \in \mathbb{N}}\left(\right.$ resp. $\left.\left(\lambda_{j+1}\right)_{j \in \mathbb{N}}\right)$ converges to this unique primal (resp. dual) solution to $\mathcal{P}_{0}$.

(ii) Suppose that there exist at least two distinct elements in $\mathcal{S}_{P}\left(\right.$ resp. $\left.\mathcal{S}_{D}\right)$, Assumption 4 holds, and $\mathcal{P}_{0}$ has the strict complementarity property, i.e. $J_{P} \cup J_{D}=\{1, \ldots, p\}$. Then the sequence $\left(x_{j+1}\right)_{j \in \mathbb{N}}$ (resp. $\left.\left(\lambda_{j+1}\right)_{j \in \mathbb{N}}\right)$ produced by Algorithm 2 converges to a primal (resp. dual) solution to $\mathcal{P}_{0}$, which is the analytic center of $\mathcal{S}_{P}\left(\right.$ resp. $\left.\mathcal{S}_{D}\right)$.

The next sections providing the proofs for the aforementioned theorems are organized as follows. After deriving some properties for the solution set to Problem (4) in Section 3.2.1, we show in Section 3.2.2 that, under the considered assumptions, the chosen line search is welldefined. Then, we derive Lemma 5, Corollary 3 and Lemma 7 in Section 3.2.3, which lead to the proof of Theorem 1 in Section 3.2.4. Section 3.3 is dedicated to the convergence analysis and convergence rate of Algorithm 1. In Section 3.3.1 we start by deriving Lemma 8 which, together with Lemmas 5 and 7 , ensures that the sufficient decrease, relative error and continuity conditions required in [27, Theorem 2.9] are satisfied. This leads to Proposition 1. We then derive Proposition 2 which directly leads to the proof of Theorem 2 in Section 3.3.2. Finally, we study the convergence of Algorithm 2 in Section 3.4 based on a Lagrangian approach. The proof for Theorem 3 is given in Section 3.4.1, the analytic center is presented in Section 3.4.2 and, finally, the proof of Theorem 4 is provided in 3.4.3.

\subsection{Well-definedness of Algorithm 1}

\subsubsection{Preliminary results}

We first derive a preliminary result about functions $\varphi_{\mu}$ and $\Psi_{\mu}$ with $\mu>0$.

Lemma 1 Under Assumption 1(iii), for every $\mu>0$, $\nabla \varphi_{\mu}$ is Lipschitz-continuous on every compact subset of $\mathcal{D}$.

Proof Let $\mathcal{K}$ be a compact subset of $\mathcal{D}$. By assumption, for every $i \in\{1, \ldots, p\}, c_{i}$ is differentiable on $\mathcal{K}$, so it is continuous on $\mathcal{K}$ and, according to the extreme value theorem, it is bounded on $\mathcal{K}$ and it attains its bounds. Thus, there exist $(\underline{c}, \bar{c}) \in]-\infty, 0\left[^{2}\right.$ such that $(\forall i \in\{1, \ldots, p\})(\forall x \in \mathcal{K}) \underline{c} \leq c_{i}(x)<\bar{c}$. Hence, for every $(x, y) \in \mathcal{K}^{2}$,

$$
\begin{aligned}
& \|\nabla \mathcal{B}(x)-\nabla \mathcal{B}(y)\| \\
& \leq \sum_{i=1}^{p} \frac{\left\|c_{i}(y) \nabla c_{i}(x)-c_{i}(x) \nabla c_{i}(y)\right\|}{\left|c_{i}(x) c_{i}(y)\right|} \\
& \leq \sum_{i=1}^{p} \frac{\left|c_{i}(y)\right|}{\underline{c}^{2}}\left\|\nabla c_{i}(x)-\nabla c_{i}(y)\right\| \\
& \quad+\frac{\left|c_{i}(y)-c_{i}(x)\right|}{\underline{c}^{2}}\left\|\nabla c_{i}(y)\right\| .
\end{aligned}
$$

In addition, by assumption, for every $i \in\{1, \ldots, p\}$, $\nabla c_{i}$ is $L_{i}$-Lipschitz continuous on $\mathcal{K}$ for some $L_{i}>0$; in particular, it is bounded by some constant $K_{i}>$ 0 . Hence, for every $i \in\{1, \ldots, p\}, c_{i}$ is $K_{i}$-Lipschitz continuous on $\mathcal{K}$ and we deduce from (9) that

$\|\nabla \mathcal{B}(x)-\nabla \mathcal{B}(y)\| \leq\left(\sum_{i=1}^{p} \frac{\bar{c} L_{i}+K_{i}^{2}}{\underline{c}^{2}}\right)\|x-y\|$.

Therefore, for every $\mu>0, \nabla \varphi_{\mu}=\nabla g+\mu \nabla \mathcal{B}$ is Lipschitz continuous on $\mathcal{K}$. 
The following lemma will be useful.

Lemma 2 [4, Theorem 4] Under Assumption 1, for every $\mu>0$ and $\tau \in \mathbb{R}$, the level set $\left\{x \in \mathcal{D} \mid \Psi_{\mu}(x) \leq \tau\right\}$ is compact.

We now prove the existence of a solution for every subproblem $\mathcal{P}_{\mu}(4)$.

Corollary 1 Under Assumption 1, for every $\mu>0$, the solution set to $\mathcal{P}_{\mu}$ is a nonempty convex and compact subset of $\mathcal{D}$.

Proof Let $\mu>0$. By assumption there exists $x_{0} \in \mathcal{D}$ such that $x_{0} \in \operatorname{dom} \Psi_{\mu}$. Let $\mathcal{S}=\left\{x \in \mathcal{D} \mid \Psi_{\mu}(x) \leq\right.$ $\left.\Psi_{\mu}\left(x_{0}\right)\right\}$. From Lemma $2, \mathcal{S}$ is compact. The set $\mathcal{S}$ is also nonempty since $x_{0} \in \mathcal{S}$, and it is convex since $\Psi_{\mu} \in \Gamma_{0}\left(\mathbb{R}^{n}\right)$ and $\mathcal{D}$ is convex. Solving $\mathcal{P}_{\mu}$ amounts to minimizing $\Psi_{\mu}$ over $\mathcal{S}$. Hence, the solution set of $\mathcal{P}_{\mu}$ is nonempty, convex and closed; it is also bounded since it is a subset of $\mathcal{S}$, which is compact.

\subsubsection{Line search}

We show in this section that our proposed line search is well-defined given our assumptions. Let $(\bar{\gamma}, \mu) \in] 0,+\infty\left[^{2}\right.$, $\theta \in] 0,1\left[\right.$ and $A \in \mathfrak{S}_{n}$. We define function $h$ such that for every $x \in \mathcal{D}$ and $l \in \mathbb{N}$,

$h(x, l)=\operatorname{prox}_{\bar{\gamma} \theta^{l} f}^{A}\left(x-\bar{\gamma} \theta^{l} A^{-1} \nabla \varphi_{\mu}(x)\right)$.

Note that, from (2), for every $x \in \mathcal{D}$ and $l \in \mathbb{N}$,

$A(x-h(x, l))-\bar{\gamma} \theta^{l} \nabla \varphi_{\mu}(x) \in \bar{\gamma} \theta^{l} \partial f(h(x, l))$.

First, we check that, in the backtracking procedure, if the stepsize tends to zero then the expression for the next iterate in Algorithm 1 converges to the current iterate.

Lemma 3 Under Assumption 1, for every $\theta \in] 0,1[$, $(\bar{\gamma}, \mu) \in] 0,+\infty\left[^{2}\right.$ and $A \in \mathfrak{S}_{n}$, if $x \in \mathcal{D}$, then

$\lim _{l \rightarrow+\infty} h(x, l)=x$.

Proof Let $l \in \mathbb{N}$ and $x \in \mathcal{D}$. Since $\operatorname{prox}_{\bar{\gamma} \theta^{l} f}^{A}$ is nonexpansive with respect to $\|\cdot\|_{A}$, it follows that

$\left\|h(x, l)-\operatorname{prox}_{\bar{\gamma} \theta^{l} f}^{A}(x)\right\|_{A} \leq \bar{\gamma} \theta^{l}\left\|A^{-1} \nabla \varphi_{\mu}(x)\right\|_{A}$.

Taking the limit in (12) we deduce that, as $l \rightarrow+\infty$, $\left\|h(x, l)-\operatorname{prox}_{\bar{\gamma} \theta^{l} f}^{A}(x)\right\|_{A} \rightarrow 0$. In addition, $A^{-1} \partial f$ is a maximally monotone operator with respect to $\|\cdot\|_{A}$. From [37, Proposition 16.27] and since $\varnothing \neq \operatorname{intdom} f \subset$ $\operatorname{dom} \partial f$, we have $\mathcal{D} \subset \overline{\mathcal{D}} \subset \operatorname{dom} f \subset \overline{\operatorname{dom} f}=\overline{\operatorname{intdom} f} \subset$ $\overline{\operatorname{dom} \partial f}$. Thus, [37, Theorem 23.48] leads to

$\lim _{l \rightarrow+\infty}\left\|\operatorname{prox}_{\bar{\gamma} \theta^{l} f}^{A}(x)-x\right\|_{A}=0$.
Finally, from the triangular inequality it follows that, as $l \rightarrow+\infty,\|h(x, l)-x\|_{A} \rightarrow 0$, hence the result since $A \in \mathfrak{S}_{n}$.

We now show that, [34, Lemma 3.6(ii)] holds for the line search.

Lemma 4 Under Assumption 1, for every $\theta \in] 0,1[$, $(\bar{\gamma}, \mu) \in] 0,+\infty\left[^{2}\right.$ and $A \in \mathfrak{S}_{n}$, if $x \in \mathcal{D} \backslash \operatorname{Argmin}(f+$ $\left.\varphi_{\mu}\right)$, then

$\frac{\bar{\gamma} \theta^{l}\left(\varphi_{\mu}(h(x, l))-\varphi_{\mu}(x)-\left\langle h(x, l)-x, \nabla \varphi_{\mu}(x)\right\rangle\right)}{\|h(x, l)-x\|_{A}^{2}} \rightarrow 0$,

as $l \rightarrow+\infty$.

Proof Take $l \in \mathbb{N}$. If $x=h(x, l)$ then, from (10), it follows that $0 \in \partial f(x)+\nabla \varphi_{\mu}(x)$ and $x$ is a minimizer of $f+\varphi_{\mu}$, which leads to a contradiction. Hence, $\|h(x, l)-x\|_{A} \neq 0$. Since $A^{-1} \partial f$ is a maximally monotone operator with respect to $\|\cdot\|_{A},[63$, Lemma 1] leads to

$\frac{\|x-h(x, l)\|_{A}}{\bar{\gamma} \theta^{l}} \leq \frac{\|x-h(x, l+1)\|_{A}}{\bar{\gamma} \theta^{l+1}}$

and $\left(\bar{\gamma} \theta^{l} /\|h(x, l)-x\|_{A}\right)_{l \in \mathbb{N}}$ is a decreasing sequence. Hence, there exists $l_{0} \in \mathbb{N}$ and $M>0$ such that

$\left(\forall l>l_{0}\right) \quad \frac{\bar{\gamma} \theta^{l}}{\|x-h(x, l)\|_{A}} \leq M$.

In addition, from Lemma 3, (11) holds. According to Assumption 1(iii), $\mathcal{D}$ is an open set, so there exist $l_{1} \geq$ $l_{0}$ and a convex subset $\mathcal{K}$ of $\mathcal{D}$ such that $x \in \mathcal{K}$ and for every $l \geq l_{1}, h(x, l) \in \mathcal{K}$. From Lemma 1 it follows that $\nabla \varphi_{\mu}$ is uniformly continuous on any compact subset of $\mathcal{K}$. From, [34, Corollary 3.4 (ii)] and the norm equivalence $\lambda_{\min }(A)^{1 / 2}\|\cdot\| \leq\|\cdot\|_{A} \leq \lambda_{\max }(A)^{1 / 2}\|\cdot\|$, where $\lambda_{\min }(A)$ and $\lambda_{\max }(A)$ are the minimal and maximal eigen values of $A$, when $l \rightarrow+\infty$ we have

$\frac{\varphi_{\mu}(h(x, l))-\varphi_{\mu}(x)-\left\langle h(x, l)-x, \nabla \varphi_{\mu}(x)\right\rangle}{\|h(x, l)-x\|_{A}} \rightarrow 0$.

Combining (13) and (14) completes the proof.

Finally, we derive Corollary 2 below which states that the line search performed at each iteration of Algorithm 1 is properly defined.

Corollary $2 \operatorname{Let}(\delta, \theta) \in] 0,1\left[^{2},(\bar{\gamma}, \mu, \bar{\nu}) \in\right] 0,+\infty\left[{ }^{3}, \underline{\nu} \in\right.$ ] $0, \bar{\nu}]$, and $x_{0} \in \mathcal{D}$. Suppose that Assumption 1 holds and that Algorithm 1 is run at iteration $k \in \mathbb{N}$. Then, $x_{k+1} \in \mathcal{D}$ and condition (5) is met for some $l \in \mathbb{N}$. 
Proof Let us prove the result by induction. First note that $x_{0} \in \mathcal{D}$. Assume that the property is satisfied at iteration $k-1$ if $k \geq 1$. Under Assumption 1, if $x_{k} \in \mathcal{D}$ is not a minimizer of $f+\varphi_{\mu}$ then we can apply Lemma 4 , which implies that the line search will stop: for any $\delta>$ 0 , (5) will be satisfied for a finite $l$. If $x_{k} \in \operatorname{Argmin}(f+$ $\varphi_{\mu}$ ) then $\tilde{x}_{k, 0}=x_{k}$ and the line search is satisfied for $l=0$. Moreover, if $x_{k+1} \notin \mathcal{D}$ then $\varphi_{\mu}\left(x_{k+1}\right)=+\infty$ and the inequality (5) could not be satisfied. Hence, $x_{k+1} \in \mathcal{D}$.

\subsubsection{Key elements for the proof of Theorem 1}

Let us first show the following sufficient decrease property regarding the values of the merit function.

Lemma 5 (Sufficient decrease) For every $(\delta, \theta) \in$ ] $\left.0,1\left[^{2},(\bar{\gamma}, \mu, \bar{\nu}) \in\right] 0,+\infty\left[^{3}, \underline{\nu} \in\right] 0, \bar{\nu}\right]$, and $x_{0} \in \mathcal{D}$, if $A s-$ sumption 1 holds, then the sequence $\left(x_{k}\right)_{k \in \mathbb{N}}$ produced by Algorithm 1 with $\epsilon=0$ satisfies, for every $k \in \mathbb{N}$, the inequality

$\Psi_{\mu}\left(x_{k+1}\right) \leq \Psi_{\mu}\left(x_{k}\right)-\frac{\nu(1-\delta)}{\bar{\gamma}}\left\|x_{k+1}-x_{k}\right\|^{2}$

Proof Let $k \in \mathbb{N}$. The stopping criterion (5) for the backtracking procedure on $\gamma_{k}$ leads to

$$
\begin{aligned}
\Psi_{\mu}\left(x_{k+1}\right) \leq \varphi_{\mu}\left(x_{k}\right)+\left\langle x_{k+1}-x_{k}, \nabla \varphi_{\mu}\left(x_{k}\right)\right\rangle \\
+\frac{\delta}{\gamma_{k}}\left\|x_{k+1}-x_{k}\right\|_{A_{k}}^{2}+f\left(x_{k+1}\right) .
\end{aligned}
$$

In addition, we have

$$
A_{k}\left(x_{k}-x_{k+1}\right)-\gamma_{k} \nabla \varphi_{\mu}\left(x_{k}\right) \in \gamma_{k} \partial f\left(x_{k+1}\right),
$$

and it follows from the definition of the subdifferential that

$$
\begin{array}{r}
\gamma_{k} f\left(x_{k}\right) \geq\left\langle A_{k}\left(x_{k}-x_{k+1}\right)-\gamma_{k} \nabla \varphi_{\mu}\left(x_{k}\right), x_{k}-x_{k+1}\right\rangle \\
+\gamma_{k} f\left(x_{k+1}\right) .
\end{array}
$$

Re-writing (17) in a more convenient form yields

$$
\begin{aligned}
& \left\langle x_{k+1}-x_{k}, \nabla \varphi_{\mu}\left(x_{k}\right)\right\rangle+f\left(x_{k+1}\right) \\
& \quad \leq f\left(x_{k}\right)-\frac{1}{\gamma_{k}}\left\|x_{k}-x_{k+1}\right\|_{A_{k}}^{2}
\end{aligned}
$$

Plugging (18) into (16) and using $\underline{\nu} \rrbracket_{n} \preceq A_{k}$ completes the proof.
Remark 3 It is worth noting that, without the assumption of existence of bounds $(\underline{\nu}, \bar{\nu})$ on matrices $\left(A_{k}\right)_{k \in \mathbb{N}}$, the proof of Lemma 5 still allows us to conclude that the sequence $\left(\Psi_{\mu}\left(x_{k}\right)\right)_{k \in \mathbb{N}}$ is decreasing. Thus, in view of Lemma 2, there exists a compact $\mathcal{K} \subset \mathcal{D}$ such that $(\forall k \in \mathbb{N}) x_{k} \in \mathcal{K}$. Therefore, by the continuity of function $c$ on $\mathcal{K}$ we deduce that there exist $\underline{c}$ and $\bar{c}$ in ]$-\infty, 0[$ such that for every $k \in \mathbb{N}$ and $i \in\{1, \ldots, p\}$, $\underline{c} \leq c_{i}\left(x_{k}\right) \leq \bar{c}<0$. This remark will be useful in Section 4 to prove that the chosen variable metrics satisfy the boundedness condition.

Before deriving a lowerbound for $\left(\gamma_{k}\right)_{k \in \mathbb{N}}$ in Lemma 6 we show that the distance between two iterates produced by Algorithm 1 tends to zero and that the iterates are bounded.

Corollary 3 Under Assumption 1, for every $(\delta, \theta) \in$ ] $\left.0,1\left[{ }^{2},(\bar{\gamma}, \mu, \bar{\nu}) \in\right] 0,+\infty\left[{ }^{3}, \underline{\nu} \in\right] 0, \bar{\nu}\right]$, and $x_{0} \in \mathcal{D}$, the sequence $\left(x_{k}\right)_{k \in \mathbb{N}}$ produced by Algorithm 1 with $\epsilon=0$ satisfies the following properties:

(i) $\lim _{k \rightarrow+\infty}\left\|x_{k+1}-x_{k}\right\|=0$;

(ii) there exists a compact $\mathcal{K} \subset \mathcal{D}$ such that $(\forall k \in \mathbb{N})$ $x_{k} \in \mathcal{K}$.

Proof (i) Summing (15) for $k=0$ to $N-1 \geq 0$ gives

$$
\begin{aligned}
\sum_{k=0}^{N-1}\left\|x_{k+1}-x_{k}\right\|^{2} & \leq \frac{\bar{\gamma}}{\underline{\nu}(1-\delta)}\left(\Psi_{\mu}\left(x_{0}\right)-\Psi_{\mu}\left(x_{N}\right)\right) \\
& \leq \frac{\bar{\gamma}}{\underline{\nu}(1-\delta)}\left(\Psi_{\mu}\left(x_{0}\right)-\underline{\Psi_{\mu}}\right)
\end{aligned}
$$

where $\Psi_{\mu}=\min _{x \in \mathbb{R}^{n}} \Psi_{\mu}(x)$. The existence of $\Psi_{\mu}$ is ensured by Corollary 1 . Letting $N$ tend to infinity gives $\sum_{k=0}^{\infty}\left\|x_{k+1}-x_{k}\right\|^{2}<+\infty$, which leads directly to the result.

(ii) From Lemma 2, the set $\left\{x \in \mathcal{D} \mid \Psi_{\mu}(x) \leq \Psi_{\mu}\left(x_{0}\right)\right\}$ is compact, and from Lemma 5 , for every $k \in \mathbb{N} x_{k}$ belongs to this set, which completes the proof.

Before deriving relative error and continuity conditions, we show that, for every barrier problem, the stepsize computed using the line search is bounded below from zero.

Lemma 6 Under Assumption 1, for every $(\delta, \theta) \in] 0,1\left[^{2}\right.$, $\left.(\bar{\gamma}, \mu, \bar{\nu}) \in] 0,+\infty\left[^{3}, \underline{\nu} \in\right] 0, \bar{\nu}\right]$, and $x_{0} \in \mathcal{D}$, there exists $\underline{\gamma}_{\mu}>0$ such that the sequence $\left(\gamma_{k}\right)_{k \in \mathbb{N}}$ generated by Algorithm 1 with $\epsilon=0$ is bounded below by $\underline{\gamma}_{\mu}$.

Proof Let $\mathcal{I}$ be the set of iterations in Algorithm 1 for which the stepsize value produced by the backtracking is strictly smaller than $\bar{\gamma}$, i.e. $\mathcal{I}=\left\{k \in \mathbb{N} \mid \gamma_{k}<\bar{\gamma}\right\}$. 
For every $k \in \mathcal{I}$ there exists an integer $l_{k}>0$ such that $\gamma_{k}=\bar{\gamma} \theta^{l_{k}}$. By applying [63, Lemma 1], we have

$(\forall k \in \mathcal{I}) \quad \frac{\left\|x_{k}-\tilde{x}_{k, l_{k}-1}\right\|_{A_{k}}}{\bar{\gamma} \theta^{l_{k}-1}} \leq \frac{\left\|x_{k}-\tilde{x}_{k, l_{k}}\right\|_{A_{k}}}{\bar{\gamma} \theta^{l_{k}}}$,

which leads to

$(\forall k \in \mathcal{I}) \quad\left\|x_{k}-\tilde{x}_{k, l_{k}-1}\right\| \leq \frac{1}{\theta}\left(\frac{\bar{\nu}}{\underline{\nu}}\right)^{\frac{1}{2}}\left\|x_{k}-x_{k+1}\right\|$.

From Corollary 3(ii), there exists a compact subset $\mathcal{K}$ of $\mathcal{D}$ such that, for every $k \in \mathbb{N}, x_{k} \in \mathcal{K}$. Let $\vartheta: \mathcal{K} \rightarrow$ $\left[0,+\infty\left[: x \mapsto d\left(x, \mathbb{R}^{n} \backslash \mathcal{D}\right)=\inf _{y \in \mathbb{R}^{n} \backslash \mathcal{D}}\|x-y\| . \vartheta\right.\right.$ is a continuous function defined on a compact set and, since $\mathcal{D}$ is open, it is positive valued. It follows from the extreme value theorem, that there exists $\eta \in] 0,+\infty[$ such that $\eta=\min _{x \in \mathcal{K}} \vartheta(x)$. For every $z \in \mathcal{K}$, let $B(z, \eta / 2)$ be the open ball with center $z$ and radius $\eta / 2$. For every $y \in \overline{B(z, \eta / 2)},\|y-z\| \leq \eta / 2<\eta \leq d\left(z, \mathbb{R}^{n} \backslash \mathcal{D}\right)$, which implies that $y \notin \mathbb{R}^{n} \backslash \mathcal{D}$, that is $y \in \mathcal{D}$. This shows that

$(\forall z \in \mathcal{K}) \quad \overline{B(z, \eta / 2)} \subset \mathcal{D}$.

On the other hand, since $\cup_{z \in \mathcal{K}} B(z, \eta / 4)$ is a cover of $\mathcal{K}$, it follows from the compactness of this latter set that there exists $\left(z_{j}\right)_{1 \leq j \leq J}$ in $\mathcal{K}$ such that

$\mathcal{K} \subset \bigcup_{j=1}^{J} B\left(z_{j}, \eta / 4\right)$

Let $\mathcal{S}=\cup_{j=1}^{J} \overline{B\left(z_{j}, \eta / 2\right)}$. It follows from (20) and (21) that this set is a compact subset of $\mathcal{D}$ including $\mathcal{K}$.

From (21), for every $k \in \mathcal{I}$, there exists $j_{k} \in\{1, \ldots, J\}$ such that

$\left\|x_{k}-z_{j_{k}}\right\|<\frac{\eta}{4}$.

On the other hand, according to Corollary 3(i), there exists $k_{0} \in \mathbb{N}$ such that

$\left(\forall k \geq k_{0}\right) \quad\left\|x_{k}-x_{k+1}\right\| \leq \theta\left(\frac{\underline{\bar{\nu}}}{\bar{\nu}}\right)^{\frac{1}{2}} \frac{\eta}{4}$.

Set $\mathcal{I}_{0}=\left\{k \in \mathcal{I} \mid k \geq k_{0}\right\}$. By applying the triangle inequality, we deduce from (19), (22), and (23) that

$\left(\forall k \in \mathcal{I}_{0}\right) \quad\left\|\tilde{x}_{k, l_{k}-1}-z_{j_{k}}\right\|<\frac{\eta}{2}$,

which shows that $\tilde{x}_{k, l_{k}-1} \in \mathcal{S}$.

Since $\varphi_{\mu}$ is convex, the following inequality holds for every $k \in \mathcal{I}_{0}$ :

$$
\begin{aligned}
& \left\langle\tilde{x}_{k, l_{k}-1}-x_{k}, \nabla \varphi_{\mu}\left(\tilde{x}_{k, l_{k}-1}\right)-\nabla \varphi_{\mu}\left(x_{k}\right)\right\rangle \geq \\
& \quad \varphi_{\mu}\left(\tilde{x}_{k, l_{k}-1}\right)-\varphi_{\mu}\left(x_{k}\right)-\left\langle\tilde{x}_{k, l_{k}-1}-x_{k}, \nabla \varphi_{\mu}\left(x_{k}\right)\right\rangle .
\end{aligned}
$$

In addition, $l_{k}$ is the smallest integer such that (5) is satisfied. Hence, (5) is not satisfied for $\tilde{x}_{k, l_{k}-1}$ and, for every $k \in \mathcal{I}_{0}$, the following holds:

$$
\begin{array}{r}
\varphi_{\mu}\left(\tilde{x}_{k, l_{k}-1}\right)-\varphi_{\mu}\left(x_{k}\right)-\left\langle\tilde{x}_{k, l_{k}-1}-x_{k}, \nabla \varphi_{\mu}\left(x_{k}\right)\right\rangle> \\
\frac{\underline{\nu} \theta \delta}{\gamma_{k}}\left\|\tilde{x}_{k, l_{k}-1}-x_{k}\right\|^{2} .
\end{array}
$$

Necessarily, $x_{k} \neq \tilde{x}_{k, l_{k}-1}$. From (24) and (25), it follows that $\left(\forall k \in \mathcal{I}_{0}\right)$,

$$
\begin{aligned}
&\left\|\tilde{x}_{k, l_{k}-1}-x_{k}\right\|\left\|\nabla \varphi_{\mu}\left(\tilde{x}_{k, l_{k}-1}\right)-\nabla \varphi_{\mu}\left(x_{k}\right)\right\|> \\
& \frac{\underline{\nu} \theta \delta}{\gamma_{k}}\left\|\tilde{x}_{k, l_{k}-1}-x_{k}\right\|^{2} .
\end{aligned}
$$

Moreover, according to Lemma $1, \nabla \varphi_{\mu}$ is Lipschitz continuous on $\mathcal{S}$. Hence, there exists $L_{\mathcal{S}}>0$ such that

$\left(\forall k \in \mathcal{I}_{0}\right) \quad \gamma_{k}>\frac{\nu \theta \delta}{L_{\mathcal{S}}}$.

In addition, $(\forall k \notin \mathcal{I}) \gamma_{k}=\bar{\gamma}$, and the set $\mathcal{I} \backslash \mathcal{I}_{0}$ has a finite number of elements. Hence, the proof is complete by setting $\underline{\gamma}_{\mu}=\min \left\{\bar{\gamma}, \frac{\nu \theta \delta}{L_{\mathcal{S}}},\left(\gamma_{k}\right)_{k \in \mathcal{I} \backslash \mathcal{I}_{0}}\right\}$.

We are now ready to identify a sequence of subgradients of $\Psi_{\mu}$ converging to zero.

Lemma 7 (Relative error condition) Under Assumption 1 , for every $(\delta, \theta) \in] 0,1\left[^{2},(\bar{\gamma}, \mu, \bar{\nu}) \in\right] 0,+\infty\left[{ }^{3}\right.$, $\underline{\nu} \in] 0, \bar{\nu}]$, and $x_{0} \in \mathcal{D}$, the sequence $\left(v_{k+1}\right)_{k \in \mathbb{N}}$ produced by Algorithm 1 when $\epsilon=0$ is such that $(\forall k \in \mathbb{N})$ $v_{k+1} \in \partial \Psi_{\mu}\left(x_{k+1}\right)$ and

$\left(\exists K_{\mu}>0\right)(\forall k \in \mathbb{N}) \quad\left\|v_{k+1}\right\| \leq K_{\mu}\left\|x_{k+1}-x_{k}\right\|$.

Proof Let $k \in \mathbb{N}$. By definition of $x_{k+1}$ and $v_{k+1}$,

$$
\begin{aligned}
v_{k+1}-\nabla \varphi_{\mu}\left(x_{k+1}\right) & =\frac{1}{\gamma_{k}} A_{k}\left(x_{k}-x_{k+1}\right)-\nabla \varphi_{\mu}\left(x_{k}\right) \\
& \in \partial f\left(x_{k+1}\right) .
\end{aligned}
$$

By definition of $\Psi_{\mu}$, it follows that $v_{k+1} \in \partial \Psi_{\mu}\left(x_{k+1}\right)$. In addition, the triangle inequality and Lemma 6 lead to

$$
\left\|v_{k+1}\right\| \leq \frac{\bar{\nu}}{\underline{\gamma}_{\mu}}\left\|x_{k}-x_{k+1}\right\|+\left\|\nabla \varphi_{\mu}\left(x_{k+1}\right)-\nabla \varphi_{\mu}\left(x_{k}\right)\right\| .
$$

From Corollary 3(ii) and Lemma 1, we know that $\left(x_{k}\right)_{k \in \mathbb{N}}$ belongs to a compact subset $\mathcal{K}$ of $\mathcal{D}$, on which $\nabla \varphi_{\mu}$ is Lipschitz-continuous for some constant $L_{\mathcal{K}}>0$. Setting $K_{\mu}=\bar{\nu} / \underline{\gamma}_{\mu}+L_{\mathcal{K}}$ completes the proof.

Remark 4 It can also be deduced from (26) that $(\forall k \in$ N) $v_{k+1}-\mu \nabla \mathcal{B}\left(x_{k+1}\right) \in \partial(f+g)\left(x_{k+1}\right)$. 


\subsubsection{Proof of Theorem 1}

In view of Lemma 7 and Corollary 3(i), for every $\epsilon>0$, there exists $k \in \mathbb{N}$ such that $\left\|v_{k+1}\right\|<\epsilon$.

\subsection{Convergence analysis of Algorithm 1}

\subsubsection{Preliminary results}

We first derive the following continuity condition.

Lemma 8 (Continuity condition) Under Assumptions 1 , let $\left.(\delta, \theta) \in] 0,1\left[{ }^{2},(\bar{\gamma}, \mu, \bar{\nu}) \in\right] 0,+\infty\left[^{3}, \underline{\nu} \in\right] 0, \bar{\nu}\right]$ and $x_{0} \in \mathcal{D}$. If the sequence $\left(x_{k}\right)_{k \in \mathbb{N}}$ is produced by Algorithm 1 with $\epsilon=0$, then there exist $\bar{x} \in \mathcal{D}$ and a subsequence $\left(x_{k_{q}}\right)_{q \in \mathbb{N}}$ such that

$\lim _{q \rightarrow+\infty} x_{k_{q}}=\bar{x}$ and $\lim _{q \rightarrow+\infty} \Psi_{\mu}\left(x_{k_{q}}\right)=\Psi_{\mu}(\bar{x})$.

Proof From Corollary 3(ii) we know that $\left(x_{k}\right)_{k \in \mathbb{N}}$ belongs to a compact subset $\mathcal{K}$ of $\mathcal{D}$. Hence, there exists a subsequence $\left(x_{k_{q}}\right)_{q \in \mathbb{N}}$ converging to an element $\bar{x} \in \mathcal{D}$. Since $\Psi_{\mu}$ is lsc, we have

$\Psi_{\mu}(\bar{x}) \leq \liminf _{q \rightarrow+\infty} \Psi_{\mu}\left(x_{k_{q}}\right)$.

Without loss of generality one can assume that $k_{0}>$ 0 . From Lemma 7 , for every $q \in \mathbb{N}, v_{k_{q}}$ belongs to $\partial \Psi_{\mu}\left(x_{k_{q}}\right)$ and $\left\|v_{k_{q}}\right\| \leq K_{\mu}\left\|x_{k_{q}}-x_{k_{q}-1}\right\|$. For every $q \in \mathbb{N}$, we have

$$
\begin{aligned}
\Psi_{\mu}\left(x_{k_{q}}\right) & \leq-\left\langle v_{k_{q}}, \bar{x}-x_{k_{q}}\right\rangle+\Psi_{\mu}(\bar{x}) \\
& \leq K_{\mu}\left\|x_{k_{q}}-x_{k_{q}-1}\right\|\left\|\bar{x}-x_{k_{q}}\right\|+\Psi_{\mu}(\bar{x}) .
\end{aligned}
$$

From Corollary 3(i), $\left\|x_{k_{q}}-x_{k_{q}-1}\right\| \rightarrow 0$ as $q \rightarrow+\infty$. Hence, taking the limit in (28) yields the following inequality

$\limsup _{q \rightarrow+\infty} \Psi_{\mu}\left(x_{k_{q}}\right) \leq \Psi_{\mu}(\bar{x})$.

Altogether (27) and (29) lead to $\lim _{q \rightarrow+\infty} \Psi_{\mu}\left(x_{k_{q}}\right)=\Psi_{\mu}(\bar{x})$.

The next result guarantees that, in the absence of stopping rule, Algorithm 1 converges to a solution to the barrier problem.

Proposition 1 Let $(\delta, \theta) \in] 0,1\left[^{2},(\bar{\gamma}, \mu, \bar{\nu}) \in\right] 0,+\infty\left[{ }^{3}\right.$, $\underline{\nu} \in] 0, \bar{\nu}]$, and $x_{0} \in \mathcal{D}$. Suppose that $\Psi_{\mu}$ is a KL function and that Assumption 1 holds. Then the sequence $\left(x_{k}\right)_{k \in \mathbb{N}}$ produced by Algorithm 1 with $\epsilon=0$ converges to a solution to $\mathcal{P}_{\mu}$ and has a finite length, i.e.

$\sum_{k=0}^{+\infty}\left\|x_{k+1}-x_{k}\right\|<+\infty$.
Proof Under Assumption 1, Lemmas 5, 7 and 8 hold. If, in addition, $\Psi_{\mu}$ is a KL function, then we can apply [27, Theorem 2.9]. Thus, $\left(x_{k}\right)_{k \in \mathbb{N}}$ converges to a critical point of $\Psi_{\mu}$ and has finite length. By convexity, every critical point of $\Psi_{\mu}$ is a global minimizer of $\Psi_{\mu}$, and a solution to $\mathcal{P}_{\mu}$.

We now show that, for a useful special case, Algorithm 1 converges linearly in terms of iterate and objective function value.

Proposition 2 Let $\mu>0$ and consider the barrier problem $\mathcal{P}_{\mu}$ associated to problem (6). Under Assumptions 1 and 3, for every $(\delta, \theta) \in] 0,1\left[^{2},(\bar{\gamma}, \bar{\nu}) \in\right] 0,+\infty\left[^{2}\right.$, $\underline{\nu} \in] 0, \bar{\nu}]$, and $x_{0} \in \mathcal{D}$, the sequence $\left(x_{k}\right)_{k \in \mathbb{N}}$ generated by Algorithm 1 with $\epsilon=0$ converges linearly to a solution $x^{*}$ to $\mathcal{P}_{\mu}$, and $\left(\Psi_{\mu}\left(x_{k}\right)\right)_{k \in \mathbb{N}}$ converges linearly to $\Psi\left(x^{*}\right)$.

Proof Let $\mu>0$. Under Assumptions 1 and 3 we can apply [43, Corollary 5.1] which states that $\Psi_{\mu}$ is a KL function with exponent $1 / 2$. The convergence of $\left(x_{k}\right)_{k \in \mathbb{N}}$ to a solution $x^{*} \in \mathcal{D}$ to $\mathcal{P}_{\mu}$ is guaranteed by Proposition 1. From Lemmas 5, 7 and 8, we can apply [22, Theorem 4(ii)]. Hence, there exist $\bar{c}>0$ and $k_{0} \in \mathbb{N}$ such that for every $k \geq k_{0}$,

$\Psi_{\mu}\left(x_{k}\right)-\Psi_{\mu}\left(x^{*}\right)=O\left(\exp \left(-\bar{c} K_{\mu}\right)^{k}\right)$

and

$\left\|x^{*}-x_{k}\right\|=O\left(\exp \left(-\bar{c} K_{\mu} / 2\right)^{k}\right)$

The linear convergence properties follow from the fact that $\exp \left(-\bar{c} K_{\mu}\right)<1$ and $\exp \left(-\bar{c} K_{\mu} / 2\right)<1$.

\subsubsection{Proof of Theorem 2}

Proposition 1 ensures the convergence of $\left(x_{k}\right)_{k \in \mathbb{N}}$ to some $x^{*}$. According to Lemma 7 , there exists $K_{\mu}>0$ such that

$$
\begin{aligned}
(\forall k \in \mathbb{N})\left\|v_{k+1}\right\| & \leq K_{\mu}\left\|x_{k+1}-x_{k}\right\| \\
& \leq K_{\mu}\left(\left\|x_{k+1}-x^{*}\right\|+\left\|x^{*}-x_{k}\right\|\right)
\end{aligned}
$$

We then deduce from Proposition 2 that there exists $\bar{c}>0$ such that $\left\|v_{k+1}\right\|=O\left(\exp \left(-\bar{c} K_{\mu} / 2\right)^{k}\right)$.

\subsection{Convergence analysis of Algorithm 2}

We are now ready to establish the convergence of Algorithm 2 to a solution to problem $\mathcal{P}_{0}$ described in (3). 
Under Assumption 1, finding a solution to $\mathcal{P}_{0}$ is equivalent to finding a saddle point of the associated Lagrangian $\mathcal{L}_{0}$, which is defined, for every $x \in \mathbb{R}^{n}$ and $\lambda \in\left[0,+\infty\left[{ }^{p}\right.\right.$, as

$\mathcal{L}_{0}(x, \lambda)=f(x)+g(x)+\lambda^{\top} c(x)$.

A point $\left(x^{*}, \lambda^{*}\right)$ with $\lambda^{*}=\left(\lambda^{*(i)}\right)_{1 \leq i \leq p}$ is a saddle point of $\mathcal{L}_{0}$ if and only if the following optimality conditions are satisfied:

$$
\left\{\begin{array}{l}
0 \in \partial f\left(x^{*}\right)+\nabla g\left(x^{*}\right)+\sum_{i=1}^{p} \lambda^{*(i)} \nabla c_{i}\left(x^{*}\right) \\
(\forall i \in\{1, \ldots, p\}) \quad \lambda^{*(i)} c_{i}\left(x^{*}\right)=0 \\
(\forall i \in\{1, \ldots, p\}) \quad \lambda^{*(i)} \geq 0 \text { and } c_{i}\left(x^{*}\right) \leq 0
\end{array}\right.
$$

We prove in the following section that the sequences $\left(x_{j}\right)_{j \in \mathbb{N}}$ and $\left(\lambda_{j+1}\right)_{j \in \mathbb{N}}$, produced by Algorithm 2, converge to a saddle point of $\mathcal{L}_{0}$. We remind that, for every $j \in \mathbb{N}$, the dual variable $\lambda_{j+1}$ is defined as follows,

$\lambda_{j+1}=\left(-\frac{\mu_{j}}{c_{i}\left(x_{j+1}\right)}\right)_{1 \leq i \leq p}$.

It is worth noting that (34) can be seen as a perturbation of condition (32).

Remark 5 In the case of affine inequality constraints, (34) can be directly derived from the Lagrangian formulation of the barrier problem. Let $j \in \mathbb{N}$ and $\mu_{j}>0$, a simple change of variable leads to the following problem, which is equivalent to $\mathcal{P}_{\mu_{j}}$,

$$
\begin{aligned}
& \underset{(x, z) \in \mathbb{R}^{n} \times \mathbb{R}^{p}}{\operatorname{minimize}} f(x)+g(x)+\mu_{j} b(z) \\
& \text { subject to } \quad z=c(x)
\end{aligned}
$$

where $b(z)=-\sum_{i=1}^{p} \ln \left(-z_{i}\right)$ if $\left.z \in\right]-\infty, 0\left[{ }^{p},+\infty\right.$ otherwise. Under Assumption 1, and when $(\forall i \in\{1, \ldots, p\})$ $c_{i}$ is affine, finding a solution to (35) is equivalent to finding a saddle point of the Lagrangian $\mathcal{L}_{\mu_{j}}$ defined, for every $x \in \mathbb{R}^{n}, z \in \mathbb{R}^{p}$ and $\lambda \in\left[0,+\infty\left[^{p}\right.\right.$, as

$\mathcal{L}_{\mu_{j}}(x, z, \lambda)=f(x)+g(x)+\mu_{j} b(z)+\lambda^{\top}(c(x)-z)$.

A point $(\bar{x}, \bar{z}, \bar{\lambda})$ is a saddle point of $\mathcal{L}_{\mu_{j}}$ if and only if the following system is satisfied.

$$
\left\{\begin{array}{l}
0 \in \partial f(\bar{x})+\nabla g(\bar{x})+\sum_{i=1}^{p} \bar{\lambda}^{(i)} \nabla c_{i}(\bar{x}) \\
(\forall i \in\{1, \ldots, p\}) \quad \bar{\lambda}^{(i)} c_{i}(\bar{x})=-\mu_{j} \\
(\forall i \in\{1, \ldots, p\}) \quad c_{i}(\bar{x})<0 \\
\bar{z}=c(\bar{x})
\end{array}\right.
$$

The definition of sequence $\left(\lambda_{j+1}\right)_{j \in \mathbb{N}}$ is then directly derived from (37).
We first show that the primal and dual sequences produced by Algorithm 2 are bounded.

Lemma 9 Under Assumptions 1 and 2 , for every $(\delta, \theta) \in$ ] $0,1\left[2,\left(\bar{\gamma}, \mu_{0}\right) \in\right] 0,+\infty\left[{ }^{2}\right.$, and $x_{0} \in \mathcal{D}$, the sequences $\left(x_{j+1}\right)_{j \in \mathbb{N}}$ and $\left(\lambda_{j+1}\right)_{j \in \mathbb{N}}$ produced by Algorithm 2 are bounded.

Proof Let $j \in \mathbb{N}$. According to Lemma $7, v_{j+1}$ belongs to $\partial \Psi_{\mu_{j}}\left(x_{j+1}\right)$. Using the definition of the subdifferential, the stopping criterion $\left\|v_{j+1}\right\| \leq \epsilon_{j}$, and the Cauchy-Schwarz inequality leads to

$$
\begin{aligned}
\Psi_{\mu_{j}}\left(x_{j+1}\right) & \leq\left\langle v_{j+1}, x_{j+1}-x_{0}\right\rangle+\Psi_{\mu_{j}}\left(x_{0}\right) \\
& \leq \epsilon_{j}\left\|x_{j+1}-x_{0}\right\|+\Psi_{\mu_{j}}\left(x_{0}\right) .
\end{aligned}
$$

Eq. (38) is equivalent to

$$
\begin{aligned}
(f+g)\left(x_{j+1}\right) \leq \mu_{j}\left(\mathcal{B}\left(x_{0}\right)-\mathcal{B}\left(x_{j+1}\right)\right) & \\
& +\epsilon_{j}\left\|x_{j+1}-x_{0}\right\|+(f+g)\left(x_{0}\right) .
\end{aligned}
$$

Moreover, since $\mathcal{B}$ is convex,

$$
\begin{aligned}
(f+g)\left(x_{j+1}\right) \leq- & \mu_{j}\left\langle\nabla \mathcal{B}\left(x_{0}\right), x_{j+1}-x_{0}\right\rangle \\
& +\epsilon_{j}\left\|x_{j+1}-x_{0}\right\|+(f+g)\left(x_{0}\right) \\
\leq( & \left(\epsilon_{j}+\mu_{j}\left\|\nabla \mathcal{B}\left(x_{0}\right)\right\|\right)\left\|x_{j+1}-x_{0}\right\| \\
& +(f+g)\left(x_{0}\right) .
\end{aligned}
$$

Assume that $\left(x_{j+1}\right)_{j \in \mathbb{N}}$ is unbounded. Then there exists a subsequence $\left(d_{q}\right)_{q \in \mathbb{N}}=\left(x_{j_{q}+1}\right)_{q \in \mathbb{N}}$ of $\left(x_{j+1}\right)_{j \in \mathbb{N}}$ such that the sequence $\left(t_{q}\right)_{q \in \mathbb{N}}=\left(\left\|x_{j_{q}+1}\right\|\right)_{q \in \mathbb{N}}$ has only strictly positive elements and satisfies

$$
\lim _{q \rightarrow+\infty} t_{q}=+\infty, \quad \lim _{q \rightarrow+\infty} \frac{d_{q}}{t_{q}}=\bar{d} \in \mathbb{R}^{n}, \quad \text { and }\|\bar{d}\|=1 .
$$

The last two equalities are derived from the compactness of the unit ball. Let $x^{*}$ be a solution to $\mathcal{P}_{0}$, take $t>0$. For every $q_{0} \in \mathbb{N}$, let $\tau_{q_{0}}=\min \left\{t_{q} \mid q \geq q_{0}\right\}$. Since $(\forall i \in\{1, \ldots, p\}) c_{i}\left(x^{*}\right) \leq 0,(\forall q \in \mathbb{N}) c_{i}\left(d_{q}\right)<0$, and $c_{i}$ is convex, $\left(\forall q \geq q_{0}\right)\left(1-\frac{\tau_{q_{0}}}{t_{q}}\right) x^{*}+\frac{\tau_{q_{0}}}{t_{q}} d_{q} \in \mathcal{D}$. By taking the limit of $\left(1-\tau_{q_{0}} / t_{q}\right) x^{*}+\left(\tau_{q_{0}} / t_{q}\right) d_{q}$ as $q \rightarrow \infty$, we obtain $x^{*}+\tau_{q_{0}} \bar{d} \in \overline{\mathcal{D}}$. In addition, for every $q \geq q_{0}$,

$$
\begin{aligned}
(f+g) & \left(\left(1-\frac{\tau_{q_{0}}}{t_{q}}\right) x^{*}+\frac{\tau_{q_{0}}}{t_{q}} d_{q}\right) \\
\leq & \left(1-\frac{\tau_{q_{0}}}{t_{q}}\right)(f+g)\left(x^{*}\right)+\frac{\tau_{q_{0}}}{t_{q}}(f+g)\left(d_{q}\right) .
\end{aligned}
$$

We deduce from (39) that

$$
\begin{array}{r}
\frac{1}{t_{q}}(f+g)\left(d_{q}\right) \leq\left(\epsilon_{j_{q}}+\mu_{j_{q}}\left\|\nabla \mathcal{B}\left(x_{0}\right)\right\|\right)\left\|\frac{d_{q}}{t_{q}}-\frac{x_{0}}{t_{q}}\right\| \\
+\frac{1}{t_{q}}(f+g)\left(x_{0}\right) .
\end{array}
$$


As $q \rightarrow+\infty$, we have $\epsilon_{j_{q}} \rightarrow 0, \mu_{j_{q}} \rightarrow 0, t_{q} \rightarrow+\infty$ and $\left\|d_{q} / t_{q}-x_{0} / t_{q}\right\| \rightarrow 1$. Hence, taking the limit in (41) leads to $\lim \sup _{q \rightarrow \infty}(f+g)\left(d_{q}\right) / t_{q} \leq 0$. Using now the lower-semicontinuity of $f+g$ and letting $q$ tend to $+\infty$ in (40) lead to

$$
\begin{aligned}
\left(\forall q_{0} \in \mathbb{N}\right) & (f+g)\left(x^{*}+\tau_{q_{0}} \bar{d}\right) \\
& \leq \liminf _{q \rightarrow \infty}(f+g)\left(\left(1-\frac{\tau_{q_{0}}}{t_{q}}\right) x^{*}+\frac{\tau_{q_{0}}}{t_{q}} d_{q}\right) \\
& \leq(f+g)\left(x^{*}\right)+\limsup _{q \rightarrow \infty} \frac{\tau_{q_{0}}}{t_{q}}(f+g)\left(d_{q}\right) \\
& \leq(f+g)\left(x^{*}\right) .
\end{aligned}
$$

Therefore, for every $q_{0} \in \mathbb{N}, x^{*}+\tau_{q_{0}} \bar{d}$ is a solution to $\mathcal{P}_{0}$. Since $\tau_{q_{0}} \rightarrow+\infty$ as $q_{0} \rightarrow+\infty$, the set of solution to $\mathcal{P}_{0}$ is unbounded. This is however in contradiction with Assumption 1(i), thus showing that $\left(x_{j+1}\right)_{j \in \mathbb{N}}$ is bounded.

Similarly, we prove that $\left(\lambda_{j}\right)_{j \in \mathbb{N}}$ is bounded. Let $j \in$ $\mathbb{N}$ and $i \in\{1, \ldots, p\}$. Since $c_{i}$ is convex, $c_{i}\left(x_{j+1}\right)<0$ and $\mu_{j}>0$, the following inequality holds:

$\mu_{j} \frac{c_{i}\left(x_{0}\right)}{c_{i}\left(x_{j+1}\right)} \leq \mu_{j}+\frac{\mu_{j}}{c_{i}\left(x_{j+1}\right)}\left\langle\nabla c_{i}\left(x_{j+1}\right), x_{0}-x_{j+1}\right\rangle$.

Summing (42) for all $i \in\{1, \ldots, p\}$ leads to

$-\left\langle c\left(x_{0}\right), \lambda_{j+1}\right\rangle \leq \mu_{j} p-\mu_{j}\left\langle\nabla \mathcal{B}\left(x_{j+1}\right), x_{0}-x_{j+1}\right\rangle$.

In addition, from Remark 4 and the definition of the subdifferential of $f+g$, we deduce that

$$
\begin{array}{r}
(f+g)\left(x_{j+1}\right) \leq-\left\langle v_{j+1}-\mu_{j} \nabla \mathcal{B}\left(x_{j+1}\right), x_{0}-x_{j+1}\right\rangle \\
+(f+g)\left(x_{0}\right) .
\end{array}
$$

Combining (43) and (44) yields

$$
\begin{array}{r}
-\left\langle c\left(x_{0}\right), \lambda_{j+1}\right\rangle \leq \mu_{j} p+(f+g)\left(x_{0}\right)-(f+g)\left(x_{j+1}\right) \\
+\epsilon_{j}\left\|x_{0}-x_{j+1}\right\| .
\end{array}
$$

Moreover, every component of $\lambda_{j+1}$ and of $-c\left(x_{0}\right)$ is strictly positive, hence

$$
\begin{array}{r}
0<-\left\langle c\left(x_{0}\right), \lambda_{j+1}\right\rangle \leq \mu_{j} p+(f+g)\left(x_{0}\right)-(f+g)\left(x^{*}\right) \\
+\epsilon_{j}\left\|x_{0}-x_{j+1}\right\|
\end{array}
$$

where $x^{*}$ is a solution to $\mathcal{P}_{0}$. Since $\left(\mu_{j}, \epsilon_{j}\right) \rightarrow(0,0)$ as $j \rightarrow+\infty$, and since $\left(x_{j+1}\right)_{j \in \mathbb{N}}$ has been shown to be bounded, we conclude that $-\left\langle c\left(x_{0}\right), \lambda_{j+1}\right\rangle$ is bounded and so is $\left(\lambda_{j+1}\right)_{j \in \mathbb{N}}$.

\subsubsection{Proof of Theorem 3}

According to Lemma 9, the sequences $\left(x_{j+1}\right)_{j \in \mathbb{N}}$ and $\left(\lambda_{j+1}\right)_{j \in \mathbb{N}}$ are bounded. Hence, there exists a subsequence $\left(x_{j_{q}+1}, \lambda_{j_{q}+1}\right)_{q \in \mathbb{N}}$ converging to some point $\left(x^{*}, \lambda^{*}\right)$. By construction, for every $q \in \mathbb{N}$ and every $i \in\{1, \ldots, p\}, c_{i}\left(x_{j_{q}+1}\right)<0$ and $0<\lambda_{j_{q}+1}^{(i)}$. Since $(\forall i \in$ $\{1, \ldots, p\}) c_{i}$ is lsc, taking the limit as $q \rightarrow+\infty$ yields (33). Moreover, by definition, $(\forall q \in \mathbb{N})(\forall i \in\{1, \ldots, p\})$ $\lambda_{j_{q}+1}^{(i)} c_{i}\left(x_{j_{q}+1}\right)=-\mu_{j_{q}}$. Since $(\forall i \in\{1, \ldots, p\}) c_{i}$ is continuous on $\overline{\mathcal{D}}$, letting $q \rightarrow+\infty$ in the previous equality leads to (32). Let $q \in \mathbb{N}$. From Remark 4, it follows that $x_{j_{q}+1}=\operatorname{prox}_{f+g}\left(x_{j_{q}+1}+v_{j_{q}+1}-\mu_{j_{q}} \nabla \mathcal{B}\left(x_{j_{q}+1}\right)\right)$. In addition, $\operatorname{prox}_{f+g}$ is nonexpansive. Hence,

$$
\begin{gathered}
\left\|x_{j_{q}+1}-\operatorname{prox}_{f+g}\left(x^{*}-\sum_{i=1}^{p} \lambda^{*(i)} \nabla c_{i}\left(x^{*}\right)\right)\right\| \\
\leq \| x_{j_{q}+1}+v_{j_{q}+1}-\mu_{j_{q}} \nabla \mathcal{B}\left(x_{j_{q}+1}\right)-x^{*} \\
+\sum_{i=1}^{p} \lambda^{*(i)} \nabla c_{i}\left(x^{*}\right) \| .
\end{gathered}
$$

By applying the triangle inequality, we deduce from (45) that

$$
\begin{aligned}
& \left\|x^{*}-\operatorname{prox}_{f+g}\left(x^{*}-\sum_{i=1}^{p} \lambda^{*(i)} \nabla c_{i}\left(x^{*}\right)\right)\right\| \\
& \leq 2\left\|x^{*}-x_{j_{q}+1}\right\|+\epsilon_{j} \\
& \quad+\sum_{i=1}^{p}\left\|\lambda_{j_{q}+1}^{(i)} \nabla c_{i}\left(x_{j_{q}+1}\right)-\lambda^{*(i)} \nabla c_{i}\left(x^{*}\right)\right\| .
\end{aligned}
$$

The sequence $\left(x_{j+1}\right)_{j \in \mathbb{N}}$ is a bounded sequence in $\mathcal{D}$. Therefore, all its cluster points belong to a compact subset of $\overline{\mathcal{D}}$. In view of Assumption 1 (iii), $\nabla c_{i}$ is thus continuous at $x^{*}$. Thus, taking the limit in (46) as $q \rightarrow$ $+\infty$ leads to

$x^{*}=\operatorname{prox}_{f+g}\left(x^{*}-\sum_{i=1}^{p} \lambda^{*(i)} \nabla c_{i}\left(x^{*}\right)\right)$,

which is equivalent to (31). Finally, $\left(x^{*}, \lambda^{*}\right)$ is a saddlepoint for the Lagrangian (30), which completes the proof.

\subsubsection{Analytic center}

When there are several primal or dual solutions, assumptions are needed to prove the uniqueness of the cluster point exhibited in Theorem 3. As shown in this section, interior point methods provide an insightful characterization for the limit point.

Let us recall that under Assumption 1, strong duality holds and the set of saddle points for the Lagrangian 
$\mathcal{L}_{0}$ is equal to $\mathcal{S}_{P} \times \mathcal{S}_{D}$ where $\mathcal{S}_{P}$ and $\mathcal{S}_{D}$ are the socalled sets of primal and dual solutions to $\mathcal{P}_{0}[62$, Section 5.4.2]. We derive the following result for these sets.

Proposition 3 Under Assumptions 1(i)-(ii), $\mathcal{S}_{P}$ and $\mathcal{S}_{D}$ are nonempty bounded convex sets.

Proof The results for $\mathcal{S}_{P}$ directly follows from Assumptions 1(i)-(ii). The convexity of $\mathcal{S}_{D}$ follows from standard results [62, Section 5.2]. The fact that $\mathcal{S}_{D}$ is not empty also follows from Assumption 1(i)-(ii). For every $\left(x^{*}, \lambda^{*}\right) \in \mathcal{S}_{P} \times \mathcal{S}_{D}$, the inequality $(f+g)\left(x^{*}\right) \leq$ $(f+g)\left(x_{0}\right)+\lambda^{* \top} c\left(x_{0}\right)$ holds for every $x_{0} \in \mathcal{D}$. If $\mathcal{S}_{D}$ is unbounded, then the right-hand side can tend to $-\infty$ which is in contradiction with $(f+g)\left(x^{*}\right)$ being a finite number. So $\mathcal{S}_{D}$ is bounded.

We also show that under an additional assumption, the sets $J_{P}$, defined in (7), and $J_{D}$, defined in (8), are nonempty.

Proposition 4 If Assumption 4 holds and if the set $\mathcal{S}_{P}\left(\right.$ resp. $\left.\mathcal{S}_{D}\right)$ contains at least two distinct elements, then the set $J_{P}$ (resp. $\left.J_{D}\right)$ is nonempty.

Proof Assume that there are at least two distinct elements $x_{1}$ and $x_{2}$ in $\mathcal{S}_{P}$. Then, for every $i \in\{1, \ldots, p\}$, $c_{i}\left(x_{1}\right) \leq 0$ and $c_{i}\left(x_{2}\right) \leq 0$. Assume that Assumption 4 holds. If the constraints are affine, i.e. $c: x \mapsto M x+m$, with $M$ an injective matrix, then there exists $i_{0} \in$ $\{1, \ldots, p\}$ such that $\left(M x_{1}\right)_{i_{0}} \neq\left(M x_{2}\right)_{i_{0}}$. There thus exists $i_{0} \in\{1, \ldots, p\}$ such that $c_{i_{0}}\left(x_{1}\right) \neq c_{i_{0}}\left(x_{2}\right)$. The same conclusion obviously holds if $i_{0} \in\{1, \ldots, p\}$ is such that $c_{i_{0}}$ is strictly convex. Hence, we have either $c_{i_{0}}\left(x_{1}\right)<0$ or $c_{i_{0}}\left(x_{2}\right)<0$, that is $i_{0} \in J_{P}$.

Assume that there are at least two distinct elements $\lambda_{1}$ and $\lambda_{2}$ in $\mathcal{S}_{D}$. The components of two elements satisfy $(\forall i \in\{1, \ldots, p\}) \lambda_{1}^{(i)} \geq 0$ and $\lambda_{2}^{(i)} \geq 0$. Since $\lambda_{1}$ and $\lambda_{2}$ are distinct there exists $i_{0} \in\{1, \ldots, p\}$ such that $\lambda_{1}^{\left(i_{0}\right)} \neq \lambda_{2}^{\left(i_{0}\right)}$. Hence, we have either $\lambda_{1}^{\left(i_{0}\right)}>0$ or $\lambda_{2}^{\left(i_{0}\right)}>0$, that is $i_{0} \in J_{D}$.

If $J_{P}$ is nonempty, we define the following quantities: for every $x \in \mathbb{R}^{n}, c_{J_{P}}(x)=\left(c_{i}(x)\right)_{i \in J_{P}}$ and $\mathcal{B}_{J_{P}}(x)=$ $-\sum_{i \in J_{P}} \ln \left(-c_{i}(x)\right)$ if $c_{J_{P}}(x)<0,+\infty$ otherwise. We also consider the following problem:

$$
\begin{aligned}
\mathcal{P}_{P}: \underset{x \in \mathbb{R}^{n}}{\operatorname{minimize}} \mathcal{B}_{J_{P}}(x) & \\
& \text { subject to } x \in \mathcal{S}_{P} \text { and } c_{J_{P}}(x)<0 .
\end{aligned}
$$

Similarly if $J_{D}$ is nonempty, we define the following quantities: for every $\lambda \in \mathbb{R}^{p}, \lambda_{J_{D}}=\left(\lambda^{(i)}\right)_{i \in J_{D}}$ and $b_{J_{D}}(\lambda)=-\sum_{i \in J_{D}} \ln \left(\lambda^{(i)}\right)$ if $\lambda_{J_{D}}>0,+\infty$ otherwise. We also consider the following problem:

$$
\begin{aligned}
\mathcal{P}_{D}: & \underset{\lambda \in \mathbb{R}^{p}}{\operatorname{minimize}} b_{J_{D}}(\lambda) \\
& \text { subject to } \lambda \in \mathcal{S}_{D} \text { and } \lambda_{J_{D}}>0 .
\end{aligned}
$$

Lemma 10 Under Assumptions 1 and 4 , if $\mathcal{S}_{P}$ (resp. $\left.\mathcal{S}_{D}\right)$ does not reduce to a singleton, then there exists a unique solution to $\mathcal{P}_{P}\left(\right.$ resp. $\left.\mathcal{P}_{D}\right)$ called the analytic center of $\mathcal{S}_{P}\left(\right.$ resp. $\left.\mathcal{S}_{D}\right)$.

Proof Assume that $\mathcal{S}_{P}$ does not reduce to a singleton. According to Proposition 3, $\mathcal{S}_{P}$ is nonempty and it thus contains at least two distinct elements. It then follows from Proposition 4 that the set $J_{P}$ is nonempty. In addition, by invoking again Proposition $3, \mathcal{S}_{P}$ is bounded and convex. Since the constraint functions $\left(c_{i}\right)_{1 \leq i \leq p}$ are convex, we deduce that the feasible set of $\mathcal{P}_{P}$ is bounded, and convex. Because of the convexity of the set $\mathcal{S}_{P}$ and the functions $\left(c_{i}\right)_{1 \leq i \leq p}$, it can be checked that this feasible set is nonempty. In addition, under Assumptions 1 and $4, \mathcal{B}_{J_{P}}$ is lsc and strictly convex, and it is finite-valued on $\left\{x \in \mathbb{R}^{n} \mid c_{J_{P}}(x)<0\right\}$. Hence, there exists a unique solution to $\mathcal{P}_{P}$.

Assume that $\mathcal{S}_{D}$ does not reduce to a singleton. It then follows from Propositions 3 and 4 that $J_{D} \neq \varnothing$. By using a similar reasoning as for $\mathcal{P}_{P}$ we deduce that there exists a unique solution to $\mathcal{P}_{D}$.

The complementary slackness property in (32) ensures that $J_{P} \cap J_{D}=\varnothing$. We say that $\mathcal{P}_{0}$ has the strict complementarity property if $J_{P} \cup J_{D}=\{1, \ldots, p\}$. Strict complementarity always holds in Linear Programming (LP) while in Quadratic Programming the concept of linear monotone complementarity can be used [64, Chapter 20].

\subsubsection{Proof of Theorem 4}

(i) The result straightforwardly follows from Theorem 3. (ii) Let $\left(x^{*}, \lambda^{*}\right)$ be a primal-dual solution to $\mathcal{P}_{0}$. Let $(\bar{x}, \bar{\lambda})$ be a cluster point of $\left(x_{j+1}, \lambda_{j+1}\right)_{j \in \mathbb{N}}$ and let $\left(x_{j_{q}+1}, \lambda_{j_{q}+1}\right)_{q \in \mathbb{N}}$ be a subsequence converging to this point. Pick $q \in \mathbb{N}$. In view of Remark 4 and (31) we have that

$v_{j_{q}+1}-\sum_{i=1}^{p} \lambda_{j_{q}+1}^{(i)} \nabla c_{i}\left(x_{j_{q}+1}\right) \in \partial(f+g)\left(x_{j_{q}+1}\right)$,

and

$-\sum_{i=1}^{p} \lambda^{*(i)} \nabla c_{i}\left(x^{*}\right) \in \partial(f+g)\left(x^{*}\right)$.

Since $f+g$ is convex, we deduce from the monotonicity of its subdifferential that

$$
\begin{aligned}
& 0 \leq\left\langle x_{j_{q}+1}-x^{*}, v_{j_{q}+1}\right\rangle \\
& -\sum_{i=1}^{p}\left\langle x_{j_{q}+1}-x^{*}, \lambda_{j_{q}+1}^{(i)} \nabla c_{i}\left(x_{j_{q}+1}\right)-\lambda^{*(i)} \nabla c_{i}\left(x^{*}\right)\right\rangle .
\end{aligned}
$$


In addition, $\left\|v_{j_{q}+1}\right\| \leq \epsilon_{j_{q}}$ and $(\forall i \in\{1, \ldots, p\}) c_{i}$ is convex. Hence, we deduce from (47) that

$$
\begin{aligned}
0 \leq\left\|x_{j_{q}+1}-x^{*}\right\| \epsilon_{j_{q}} & +\sum_{i=1}^{p} \lambda^{*(i)}\left(c_{i}\left(x_{j_{q}+1}\right)-c_{i}\left(x^{*}\right)\right) \\
& +\sum_{i=1}^{p} \lambda_{j_{q}+1}^{(i)}\left(c_{i}\left(x^{*}\right)-c_{i}\left(x_{j_{q}+1}\right)\right) .
\end{aligned}
$$

From (32) and (34), $\left\langle\lambda^{*}, c\left(x^{*}\right)\right\rangle=0$ and, for every $i \in$ $\{1, \ldots, p\}, \lambda_{j_{q}+1}^{(i)} c_{i}\left(x_{j_{q}+1}\right)=-\mu_{j_{q}}$. Hence,

$0 \leq\left\|x_{j_{q}+1}-x^{*}\right\| \frac{\epsilon_{j_{q}}}{\mu_{j_{q}}}+p-\sum_{i=1}^{p}\left(\frac{\lambda^{*(i)}}{\lambda_{j_{q}+1}^{(i)}}+\frac{c_{i}\left(x^{*}\right)}{c_{i}\left(x_{j_{q}+1}\right)}\right)$.

Note that $\left(\forall i \notin J_{P}\right) c_{i}\left(x^{*}\right)=0$ and $\left(\forall i \notin J_{D}\right) \lambda^{*(i)}=$ 0 . If $J_{P}$ (resp. $J_{D}$ ) is nonempty, we can then choose $x^{*}\left(\right.$ resp. $\left.\lambda^{*}\right)$ such that $c_{J_{P}}\left(x^{*}\right)<0$ (resp. $\left.\lambda_{J_{D}}^{*}>0\right)$. Consequently, by using Assumption 2, as $q \rightarrow+\infty$,(48) becomes

$\sum_{i \in J_{D}} \frac{\lambda^{*(i)}}{\bar{\lambda}^{(i)}}+\sum_{i \in J_{P}} \frac{c_{i}\left(x^{*}\right)}{c_{i}(\bar{x})} \leq p$,

where we necessarily have

$\left(\forall i \in J_{D}\right) \bar{\lambda}^{(i)}>0$ and $\left(\forall i \in J_{P}\right) c_{i}(\bar{x})<0$.

Because of the strict complementarity, there are exactly $p$ positive terms in the left-hand side of (49). Therefore, we can apply the arithmetic-geometric mean inequality which leads to

$$
\left(\prod_{i \in J_{D}} \frac{\lambda^{*(i)}}{\bar{\lambda}^{(i)}}\right)\left(\prod_{i \in J_{P}} \frac{c_{i}\left(x^{*}\right)}{c_{i}(\bar{x})}\right) \leq 1,
$$

with the convention that, if $J_{P}$ (resp. $J_{D}$ ) is empty, the corresponding product is equal to 1 . Since Theorem 3 states that $(\bar{x}, \bar{\lambda})$ is a primal-dual solution to $\mathcal{P}_{0}$ and because $(\bar{x}, \bar{\lambda})$ satisfies (50), (51) also holds when either $\lambda^{*}=\bar{\lambda}$ or $x^{*}=\bar{x}$. Consequently,

$$
\prod_{i \in J_{P}}\left(-c_{i}\left(x^{*}\right)\right) \leq \prod_{i \in J_{P}}\left(-c_{i}(\bar{x})\right) \text { and } \prod_{i \in J_{D}} \lambda^{*(i)} \leq \prod_{i \in J_{D}} \bar{\lambda}^{(i)} .
$$

If there exist at least two distinct elements in $\mathcal{S}_{P}$ (resp. $\mathcal{S}_{D}$ ) then, from Propositions 3 and $4, J_{P}$ (resp. $J_{D}$ ) is nonempty. It follows from (52) that $\bar{x}(\operatorname{resp} . \bar{\lambda})$ is a solution to $\mathcal{P}_{P}\left(\right.$ resp. $\left.\mathcal{P}_{D}\right)$. In turn, Lemma 10 guarantees that $\mathcal{P}_{P}\left(\right.$ resp. $\left.\mathcal{P}_{D}\right)$ has a unique solution. Thus, there exists a unique cluster point for the primal (resp. dual) sequence and $\left(x_{j+1}\right)_{j \in \mathbb{N}}\left(\right.$ resp. $\left.\left(\lambda_{j+1}\right)_{j \in \mathbb{N}}\right)$ converges to the analytic center of $\mathcal{S}_{P}\left(\operatorname{resp} . \mathcal{S}_{D}\right)$.

\section{Numerical experiments}

The good performance in terms of convergence speed of PIPA are illustrated in this section on two largescale image processing applications, namely hyperspectral unmixing and texture-geometry image reconstruction $^{2}$. We also demonstrate that introducing a variable metric in PIPA can lead to a significant reduction of its computational time. All computational times are given for experiments run on Matlab 2018b on an Intel Xeon CPU E5-1650 at frequency 3.20GHz. Our code is available online ${ }^{3}$.

\subsection{Hyperspectral unmixing}

Hyperspectral imaging devices are remote sensing systems that acquire emitting light spectrum of a distant scene, here modeled as a 2D image [67]. Let $s$ and $r$ be respectively the number of acquired spectral bands and pixels of the image, and let $Y \in \mathbb{R}^{s \times r}$ denote the measured hyperspectral cube. Assume that we have access to a library $S \in \mathbb{R}^{s \times q}$, where each column of $S$ contains the spectral signatures of one material (or endmember) among $q$ that are expected to be present in the scene. The proportion or abundance of every material in every pixel is described through the abundance matrix $X \in \mathbb{R}^{q \times r}$. The following linear model is frequently used to relate the data, the endmembers and the abundances, when there is no microscopic interaction between the materials:

$Y=S X+w$,

with $w \in \mathbb{R}^{s \times r}$ a realization of an additive white Gaussian noise. The estimation of $X$ from $Y$ and $S$ is an inverse problem called unmixing [68]. Following [69, 9], we propose to formulate the following constrained minimization problem to perform the unmixing task,

$$
\begin{aligned}
\underset{X \in \mathbb{R}^{q \times r}}{\operatorname{minimize}} & \frac{1}{2}\|Y-S X\|_{2}^{2}+\kappa \sum_{i=1}^{q}\left\|\left(W X_{i}\right)_{\mathrm{d}}\right\|_{1} \\
\text { subject to } & (\forall j \in\{1, \ldots, r\}) \quad \sum_{i=1}^{q} X_{i, j} \leq 1 \\
& (\forall i \in\{1, \ldots, q\})(\forall j \in\{1, \ldots, r\}) \quad X_{i, j} \geq 0,
\end{aligned}
$$

where $\|\cdot\|_{2}$ denotes the Frobenius norm, $X_{i} \in \mathbb{R}^{r}$ with $i \in\{1, \ldots, q\}$ is the $i^{\text {th }}$ line of the abundance matrix $X$, $W \in \mathbb{R}^{r \times r}$ is a wavelet decomposition operator, $\left\|(\cdot)_{\mathrm{d}}\right\|_{1}$ is the $\ell_{1}$-norm of the detail wavelet coefficients, and

\footnotetext{
2 Preliminary results regarding the use of proximal interior point methods in these applicative contexts can be found in our previously published communications [65, 66].

3 https://github.com/mccorbineau/PIPA
} 
$\kappa \geq 0$ is a regularization parameter. It is worth noting that the linear constraints account for the atmospheric absorption [70] since, for every pixel, the sum of all fractional abundances may be less than one. Moreover, the wavelet-based penalization allows us to enforce useful spatial regularity on the sought abundance maps [58]. From this point forward, the vectorizations of $X \in \mathbb{R}^{q \times r}$ and $Y \in \mathbb{R}^{s \times r}$, in lexicographic order, are denoted by $x \in \mathbb{R}^{n}$ with $n=q r$ and $y \in \mathbb{R}^{s r}$, respectively, and $\otimes$ denotes the Kronecker product. Problem (53) can thus be re-written as in (3), with $p=n+r$ and $\left(\forall x \in \mathbb{R}^{n}\right)$ $g(x)=1 / 2\left\|y-\left(\mathbb{\square}_{r} \otimes S\right) x\right\|^{2}, f(x)=\kappa \sum_{i=1}^{q}\left\|\left(W P_{i} x\right)_{\mathrm{d}}\right\|_{1}$, $(\forall i \in\{1, \ldots, q\}) P_{i} \in \mathbb{R}^{r \times n}$ is a decimation matrix such that $P_{i} x=X_{i}$, and $c(x)=M x+m$ with

$M=\left(\begin{array}{c}\mathbb{\square}_{r} \otimes \mathbf{1}_{q}^{\top} \\ -\mathbb{\square}_{n}\end{array}\right)$ and $m=\left(\begin{array}{c}-\mathbf{1}_{r} \\ \mathbf{0}_{n}\end{array}\right)$

where $\mathbf{1}_{r}=(1, \ldots, 1)^{\top} \in \mathbb{R}^{r}$ and $\mathbf{0}_{n}=(0, \ldots, 0)^{\top} \in$ $\mathbb{R}^{n}$. The resulting minimization problem satisfies Assumptions 1, 3 and 4. Hence, Theorems 1, 2 and 3 regarding convergence and convergence rate of Algorithm 1 hold. In addition, in the considered example, the rank of $S$ is equal to $q$, so there exists a unique solution to (53) and Theorem 4(i) holds.

\subsubsection{Realistic data simulation and test configuration}

In order to simulate $Y$, we make use of the $\operatorname{Urban}^{4}$ dataset, which provides the spectral signatures and abundance maps for $q=6$ materials in $s=162$ spectral bands. We consider images of size $r=256 \times 256$. The product of the spectral library and attenuated abundance map is corrupted with an additive white Gaussian noise with a standard deviation of 0.06 . Let the signal-to-noise ratio be defined as

$\mathrm{SNR}=20 \log _{10}(\|\bar{x}\| /\|x-\bar{x}\|)$,

where $\bar{x}$ is the ground-truth for $x$. In addition, for each material $i \in\{1, \ldots, q\}$, the signal-to-noise ratio of its associated abundance map $X_{i} \in \mathbb{R}^{r}$ is

$\mathrm{SNR}_{i}=20 \log _{10}\left(\left\|\bar{X}_{i}\right\| /\left\|X_{i}-\bar{X}_{i}\right\|\right)$.

The regularization weight $\kappa$ is tuned by a grid search so as to reach the largest SNR, in that case $\kappa=0.01$. Regarding the operator $W$, we selected an orthogonal Daubechies 4 wavelet decomposition performed over 2 resolution levels. As for the variable metric, we consider two cases: the proposed method without variable metric (taken as the identity matrix), which is referred to as PIPA; and PIPA-VM, which denotes the case when, following the strategy in [71], for every $j \in \mathbb{N}$, the variable

4 www.escience.cn/people/feiyunZHU/Dataset_GT.html metric is chosen as the Hessian of $\varphi_{\mu_{j}}$. The proximity operator of the regularization term in the variable metric is computed numerically using the algorithm in [40].

Let us now discuss the boundedness condition required for the variable metrics in PIPA-VM. For every $x \in \mathcal{D}$, we have

$\varphi_{\mu}(x)=\frac{1}{2}\left\|y-\left(\mathbb{\natural}_{r} \otimes S\right) x\right\|^{2}-\mu \sum_{i=1}^{p} \ln \left(-M_{i}^{\top} x-m_{i}\right)$,

where $M_{i} \in \mathbb{R}^{n}$ (resp. $m_{i} \in \mathbb{R}$ ) is the $i^{\text {th }}$ line (resp. component) of $M$ (resp. of $m$ ). For every $\mu>0$ and $x \in \mathcal{D}$, the Hessian of $\varphi_{\mu}$ at $x$ is equal to

$\nabla^{2} \varphi_{\mu}(x)=\rrbracket_{r} \otimes\left(S^{\top} S\right)+\mu \sum_{i=1}^{p} \frac{M_{i} M_{i}^{\top}}{\left(M_{i}^{\top} x+m_{i}\right)^{2}}$

Finally, in view of Remark 3, we deduce that there exist $\underline{c}$ and $\bar{c}$ in $]-\infty, 0[$ such that, for every $k \in \mathbb{N}$ and every $i \in\{1, \ldots, p\}, \underline{c} \leq M_{i}^{\top} x_{k}+m_{i} \leq \bar{c}<0$. Since the rank of $M$ is equal to $n$, for every $\mu>0$, the aforementioned variable metrics are bounded from below and above by strictly positive constants, as required in Algorithm 1.

In order to satisfy Assumption 2, we choose the barrier parameter and precision sequences as follows,

$(\forall j \in \mathbb{N}) \epsilon_{j}=\bar{\epsilon} \frac{\mu_{j}}{\zeta^{j}}$, and $\mu_{j+1}=\frac{\mu_{j}}{\rho_{j}}$,

where $\rho_{j} \geq \rho>1, \zeta>1$ and $\bar{\epsilon}>0$. Regarding PIPA, we take $\bar{\epsilon}=10^{3}, \mu_{0}=1, \rho_{j}=1.5$ for every $j \in \mathbb{N}$ and $\zeta=1+10^{-5}$. For PIPA-VM we choose $\bar{\epsilon}=10^{5}$, $\mu_{0}=0.01, \zeta=1+10^{-5}$ and, to avoid numerical instabilities when $\mu_{j}$ is very small, we pick $\rho_{j}=1.5$ for every $j \in \mathbb{N}$ such that $\mu_{j} \geq 10^{-6}$, and decrease it gradually: if $4 \times 10^{-9} \leq \mu_{j}<10^{-6}$ then $\rho_{j}=1.1$, if $10^{-12} \leq \mu_{j}<4 \times 10^{-9}$ then $\rho_{j}=1.01$, and finally, if $\mu_{j}<10^{-12}$, then $\rho_{j}=1.001$.

We compare PIPA and PIPA-VM with three state-ofthe-art convex optimization algorithms: the alternating direction method of multipliers (ADMM) [72, 69], the Condat-Vũ primal-dual splitting algorithm (PDS) in $[73,74]$, and the generalized forward-backward splitting algorithm (GFBS) [75]. We also implement preconditioned versions of ADMM and GFBS, which are referred to as ADMM-VM and GFBS-VM, respectively. ADMM-VM is based on [76, Algorithm 2], where the metrics are taken constant as in [76, Example 3.4]. Regarding GFBS-VM, we implement [77, Algorithm 1] with a modified metric based on the Hessian of the data-fitting term in (53). 


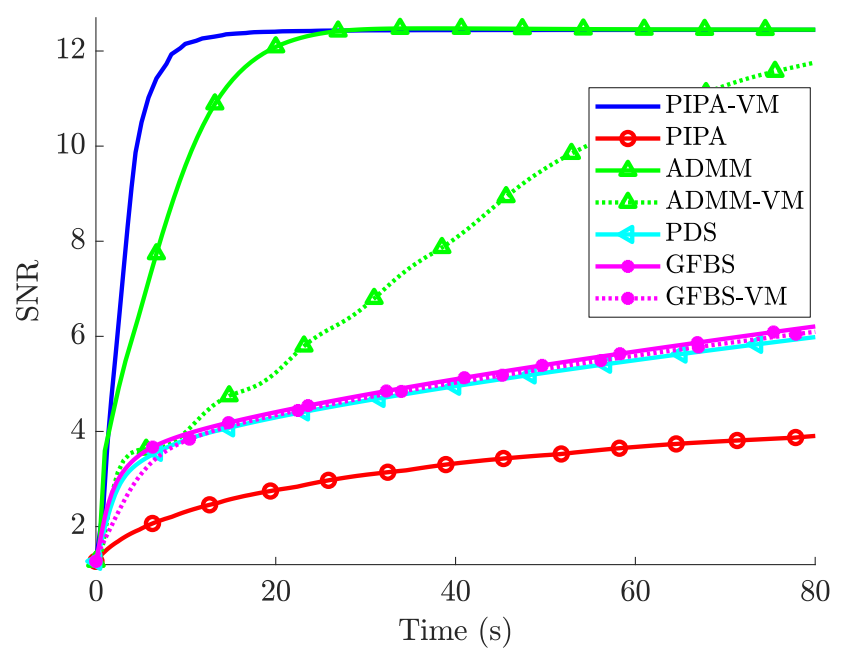

Fig. 1 SNR as a function of time.

\subsubsection{Results}

The solution to (53) with $\kappa=0$, i.e. without regularization, can be obtained with the primal-dual interior point method from [9]. It is referred to as IPLS and yields $\mathrm{SNR}=11.02 \mathrm{~dB}$, whereas solving the same problem with $\kappa=0.01$ leads to a better reconstruction with $\mathrm{SNR}=12.45 \mathrm{~dB}$, illustrating the benefits of regularizing in this example. Figure 1 shows that the SNR increases faster with PIPA-VM than with the four other algorithms. Moreover, it can be clearly seen in this figure that PIPA-VM exhibits a much faster convergence than PIPA, which demonstrates the advantage of using a variable metric in this example. The SNR obtained for each material after running PIPA-VM and ADMM for $11 \mathrm{sec}$ can be found in Table 1. Remark that we did not provide the results for PIPA, PDS, GFBS, ADMMVM and GFBS-VM, since they were outperformed by PIPA-VM and ADMM as shown in Figure 1. For 5 out of 6 endmembers, the SNR of the abundance maps obtained with PIPA-VM after 11 sec is better than for ADMM, and for all materials, PIPA-VM gives better results after $11 \mathrm{sec}$ than the non-regularized solution IPLS.

\begin{tabular}{rccc}
\hline & IPLS & ADMM & PIPA-VM \\
\hline Asphalt & 10.12 & 7.40 & $\mathbf{1 1 . 3 1}$ \\
Grass & 11.21 & 11.37 & $\mathbf{1 2 . 2 5}$ \\
Tree & 11.86 & 12.45 & $\mathbf{1 3 . 0 4}$ \\
Roof & 14.91 & 15.08 & $\mathbf{1 5 . 2 7}$ \\
Metal & 4.90 & $\mathbf{7 . 2 5}$ & 7.12 \\
Dirt & 13.68 & 12.34 & $\mathbf{1 4 . 5 2}$ \\
\hline
\end{tabular}

Table 1 Signal-to-noise ratio $\left(\mathrm{SNR}_{i}\right)_{1 \leq i \leq 6}$ (in $\left.\mathrm{dB}\right)$ for each material after $11 \mathrm{sec}$.

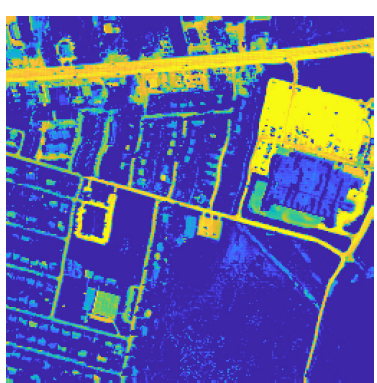

(a)



(c)

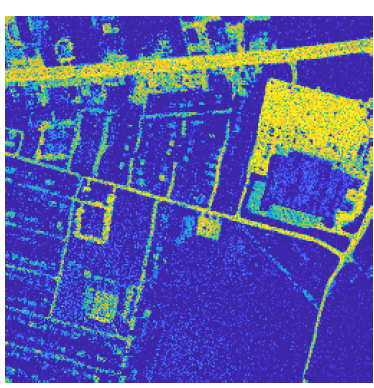

(b)

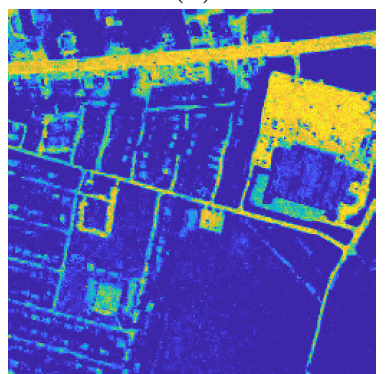

(d)
Fig. 2 Abundance map of Asphalt road: (a) ground-truth, (b) IPLS solution, visual results after running (c) ADMM and (d) PIPA-VM for 11 sec.

Visual results for Asphalt and Dirt materials are displayed in Figures 2 and 3. One can notice that, after running all algorithms for $11 \mathrm{sec}$, the abundance maps produced by PIPA-VM for these two materials are visually more satisfactory than the ones obtained with ADMM, while the non-regularized solution IPLS is significantly noisy.

In order to evaluate the algorithms based on their pointwise convergence, we let them run for a very large number of iterations and compute the relative distance between the current iterate and the solution $x_{\infty}$. As one can see on Figure 4, the sequence generated by PIPA-VM converges faster to the solution than the iterates produced by the other algorithms. It is finally worth noticing that, although an inexact computation of the proximity operator is performed in PIPA-VM, the method appears to be robust to the error generated by this approximation.

\subsection{Joint geometry-texture decomposition and reconstruction}

Various problems in image processing and computer vision can be formulated as the decomposition of a natural image into texture and geometry components. One can mention, for instance, texture segmentation [78], classification [79], or digital inpainting [80]. In the following, we will denote by $x^{t} \in \mathbb{R}^{r}$ and $x^{g} \in \mathbb{R}^{r}$, the texture and geometry components of a natural image 




(a)

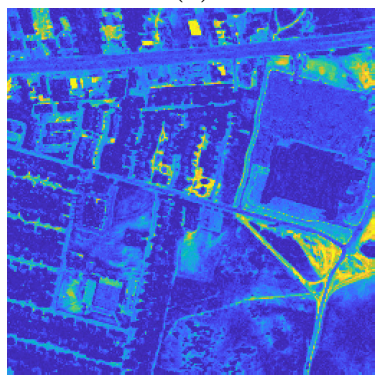

(c)

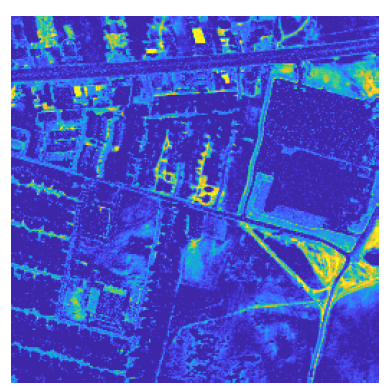

(b)

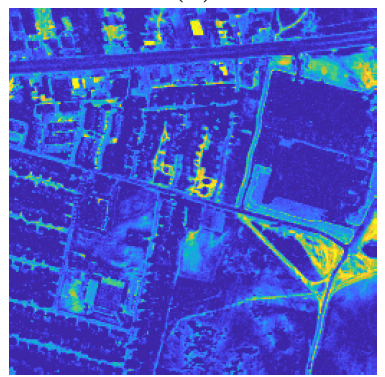

(d)
Fig. 3 Abundance map of Dirt: (a) ground-truth, (b) IPLS solution, visual results after running (c) ADMM and (d) PIPA-VM for 11 sec.

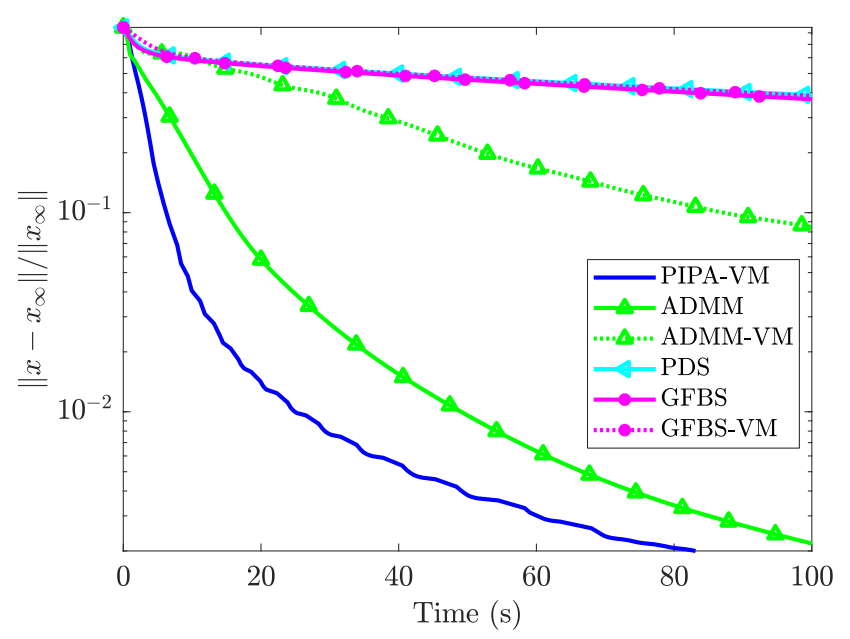

Fig. 4 Relative distance from current iterate to limit point as a function of time.

$x^{t+g} \in \mathbb{R}^{r}$, so that $x^{t+g}=x^{t}+x^{g}$. The geometry $x^{g}$ represents a piecewise smooth version of the image, and can be extracted by using the total variation semi-norm [13]. The texture highlights local components with higher spatial frequencies. Depending on the considered application, different texture models can be found in the literature, based on wavelet decompositions [81] or on the Hölder exponent [82], to name only a few. Here, we will focus on images in which the texture is located near the boundaries of different objects, as it can happen for instance in material image analysis, where the samples are subject to erosion and microporosity. Therefore, we will rely on the Laplacian detector for texture extraction, as the latter is known to be useful for edge and blob detection [83].

X-Ray Computed Tomography (CT), a fast non-destructive scanning technique [84], is frequently used to acquire images from material samples. The acquisition process in $\mathrm{CT}$ can be modeled through the discrete Radon projection operator $H \in \mathbb{R}^{q \times r}$, with $r$ the number of pixels and $q$ the number of measurements. Matrix $H$ is high-dimensional and ill-conditioned. Reconstructing the image from the measured data $y \in \mathbb{R}^{q}$ (also called sinogram) is a challenging inverse problem [85], the resolution of which can introduce a bias in subsequent image processing tasks, such as the classification of material components [86]. Therefore, we propose to perform jointly two tasks: the reconstruction and the geometry-texture decomposition. We show that this decomposition can be performed in a reasonable time with PIPA.

More precisely, we consider the following variational formulation:

$$
\begin{array}{cl}
\underset{\left(x^{t}, x^{g}\right) \in \mathbb{R}^{r} \times \mathbb{R}^{r}}{\operatorname{minimize}} & \frac{1}{2}\left\|F x^{t}\right\|^{2}+\kappa \operatorname{TV}\left(x^{g}\right) \\
\text { subject to } & x^{t}+x^{g} \in\left[x_{\min }, x_{\max }\right]^{r} \\
& x^{t} \in[-\alpha, \alpha]^{r} \\
& \left\|H\left(x^{t}+x^{g}\right)-y\right\|_{\infty} \leq \chi
\end{array}
$$

where $x_{\min }=0$ and $x_{\max }=1$ are the minimal and maximal pixel intensity values, $\alpha>0$ is a range value parameter for the texture, $\kappa>0$ is a regularization parameter, $\chi>0$ is an upperbound on the measurement uncertainty, TV denotes the isotropic total variation semi-norm with (zero) Dirichlet boundary conditions. Moreover, $F=\mathbb{q}_{r}-\Delta \in \mathbb{R}^{r \times r}$ where $\Delta \in \mathbb{R}^{r \times r}$ is the Laplacian associated with the following $2 \mathrm{D}$ kernel padded with circulant assumption:

$\left(\begin{array}{ccc}0 & 1 & 0 \\ 1 & -4 & 1 \\ 0 & 1 & 0\end{array}\right)$

The first term in the objective function enforces edge detection in the texture, while the geometry is made piecewise smooth thanks to the total variation regularization. The first set of constraints represents bounds on the pixel values of the natural image. The texture, which is supposed to capture small variations in the image, is modeled as a zero-centered component in the second set of constraints, where we take $\alpha=x_{\max } / 3$. The last constraint is the data-fit term, which can be decomposed into $2 \times q$ linear inequalities. Hence, the constraints can be reformulated as $M x+m \in]-\infty, 0]^{p}$ where $x=\left[\left(x^{t}\right)^{\top},\left(x^{g}\right)^{\top}\right]^{\top}, M \in \mathbb{R}^{p \times n}, m \in \mathbb{R}^{p}, p=$ 
$2(q+2 r), n=2 r$,

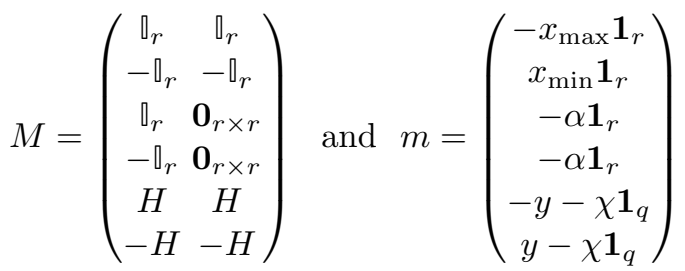

with $\mathbf{0}_{r \times r}$ the zero matrix in $\mathbb{R}^{r \times r}$.

As in the previous example, (55) is an instance of Problem (3) where $\left(\forall x \in \mathbb{R}^{n}\right) f(x)=\kappa \mathrm{TV}\left(P_{g} x\right)$ and $g(x)=$ $1 / 2\left\|F P_{t} x\right\|^{2}$, with $P_{t}=\left(\begin{array}{ll}\mathbb{Q}_{r} & \mathbf{0}_{r \times r}\end{array}\right)$ and $P_{g}=\left(\begin{array}{ll}\mathbf{0}_{r \times r} & \mathbb{q}_{r}\end{array}\right)$. It can be noted that Assumptions 1 and 4 are satisfied. Thus, Theorems 1 and 3 hold. Since Assumption 3 does not hold, Theorem 2 does not apply here. The solution to (55) is not necessarily unique. Although the strict complementarity required to apply Theorem 4 is difficult to check, the convergence of PIPA to a single cluster point was observed in practice.

\subsubsection{Initialization}

In order to find an initial point that satisfies strictly the constraints, we set $x^{t}$ to zero. Following the method in [62, Chap. 11.4], we solve the minimization problem below to initialize $x^{g}$,

$$
\begin{array}{ll}
\underset{\left(s, x^{g}\right) \in \mathbb{R} \times \mathbb{R}^{r}}{\operatorname{minimize}} & s \\
\text { subject to } & s \geq 0, x^{g} \in\left[x_{\min }, x_{\max }\right]^{r} \\
& \left\|H x^{g}-y\right\|_{\infty} \leq \chi+s,
\end{array}
$$

where $s \geq 0$ is the maximal infeasibility. The groundtruth natural image $\bar{x}^{t+g}$ satisfies $\left\|H \bar{x}^{t+g}-y\right\|_{\infty}<\chi$ so that the solution to (56) is reached for $s=0$. Problem (56) is a linear programming problem, that we solve by using the code available online ${ }^{5}$ for the primaldual interior point approach used in [62, Chap. 11.4]. This iterative algorithm generates iterates that belong to $] x_{\min }, x_{\max }{ }^{r}$ so that, in our numerical experiments, we are able to find a strictly feasible initial point in a reasonable time.

\subsubsection{Variable metric and hyperparameters}

Let $\mu_{j}>0$ and $k \in \mathbb{N}$. For every $x \in \mathcal{D}$, the Hessian of $\varphi_{\mu_{j}}$ at $x \in \mathbb{R}^{n}$ is equal to

$\nabla^{2} \varphi_{\mu_{j}}(x)=\left(\begin{array}{cc}F^{\top} F+\mu_{j} D_{3}(x)+G(x) & G(x) \\ G(x) & G(x)\end{array}\right)$

where

$G(x)=\mu_{j}\left(D_{1}(x)+H^{\top} D_{2}(x) H\right)$.

\footnotetext{
5 https://web.stanford.edu/ boyd/cvxbook/cvxbook_ examples/chap11/
}

Hereabove, $D_{1}(x) \in \mathbb{R}^{r \times r}, D_{2}(x) \in \mathbb{R}^{q \times q}$, and $D_{3}(x) \in$ $\mathbb{R}^{r \times r}$ are diagonal matrices with respective vectors of diagonal elements $d_{1}(x) \in \mathbb{R}^{r}, d_{2}(x) \in \mathbb{R}^{q}$, and $d_{3}(x) \in$ $\mathbb{R}^{r}$, defined as

$$
\begin{aligned}
(\forall i \in\{1, \ldots, r\}) \quad\left(d_{1}(x)\right)^{(i)} & =\left(\left(x^{t+g}\right)^{(i)}-x_{\min }\right)^{-2} \\
& +\left(x_{\max }-\left(x^{t+g}\right)^{(i)}\right)^{-2}
\end{aligned}
$$

$$
\begin{gathered}
(\forall j \in\{1, \ldots, q\}) \quad\left(d_{2}(x)\right)^{(j)}=\left(\left(H x^{t+g}-y\right)^{(j)}+\chi\right)^{-2} \\
+\left(\left(y-H x^{t+g}\right)^{(j)}+\chi\right)^{-2} \\
(\forall i \in\{1, \ldots, r\}) \quad\left(d_{3}(x)\right)^{(i)}=\left(\left(x^{t}\right)^{(i)}+\alpha\right)^{-2} \\
+\left(\alpha-\left(x^{t}\right)^{(i)}\right)^{-2}
\end{gathered}
$$

Given the huge size and ill-conditioning of $H$, the inversion of $\nabla^{2} \varphi_{\mu_{j}}(x)$ is hardly feasible. Hence, instead of using the full Hessian of $\varphi_{\mu_{j}}$ for the variable metric as in Section 4.1, we propose to use an upper-bound of it, i.e. $A_{k} \in \mathfrak{S}_{n}$ such that $A_{k}-\nabla^{2} \varphi_{\mu_{j}}\left(x_{k}\right)$ also belongs to $\mathfrak{S}_{n}$. We propose to majorize $\mu_{j} D_{3}(x)$ by $\beta(x) \rrbracket_{r}$ where

$\beta(x)=\max _{1 \leq i \leq r} \mu_{j}\left(d_{3}(x)\right)^{(i)}$

For $H^{\top} D_{2}(x) H$, we propose to follow the strategy in [87] and upper-bound it by the diagonal matrix $D_{4}(x) \in$ $\mathbb{R}^{r \times r}$ with vector of diagonal elements $P^{\top} d_{2}(x)$, where $P \in \mathbb{R}^{q \times r}$ is such that for every $i \in\{1, \ldots, r\}$ and $j \in\{1, \ldots, q\}$,

$P_{j, i}=H_{j, i} \sum_{s=1}^{r} H_{j, s}$.

This leads to the following variable metric in Algorithm 1:

$(\forall k \in \mathbb{N}) A_{k}=\left(\begin{array}{cc}F^{\top} F+\beta\left(x_{k}\right) \rrbracket_{r}+D\left(x_{k}\right) & D\left(x_{k}\right) \\ D\left(x_{k}\right) & D\left(x_{k}\right)\end{array}\right)$

where

$D\left(x_{k}\right)=\mu_{j}\left(D_{1}\left(x_{k}\right)+D_{4}\left(x_{k}\right)\right)$.

Since $D\left(x_{k}\right)$ is diagonal, the operator $A_{k}$ is straightforward to invert using the Schur formula. In addition, similarly to Section 4.1, we deduce from Remark 3 that matrix (57) satisfies the boundedness condition required in Algorithm 1. In order to compute the proximity operator of $f$ in such variable metric, we use [88, alg. 2]. 

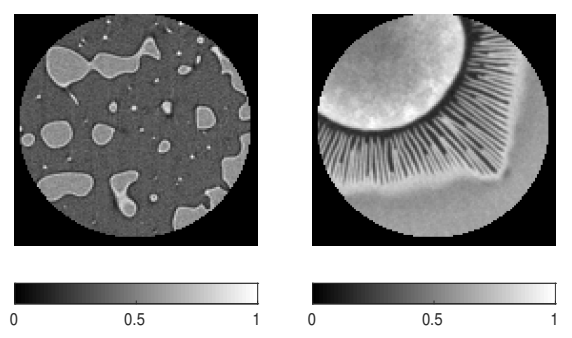

Fig. 5 Natural images: (left) phase-separated barium borosilicate glass sample, imaged at the ESRF synchrotron (courtesy of David Bouttes), (right) mushroom Agaricus bisporus (courtesy of DigiMorph.org, The University of Texas High-Resolution X-ray CT Facility (UTCT), and NSF grant IIS-0208675).

Regarding the hyperparameters in the proposed method, in order to satisfy Assumption 2, as in the previous example, we take sequences of the form (54) with $\mu_{0}=$ $10^{-3}, \zeta=1+10^{-5}, \bar{\epsilon}=8.3 \times 10^{3}$ and $\rho_{j}=1.1$ for every $j \in \mathbb{N}$.

\subsubsection{Test settings}

We perform the joint reconstruction and decomposition of two high-quality scans, referred to as Glass and Agaricus, which are displayed in Figure 5. These images are of size $r=128 \times 128$. The discrete Radon operator $H$ models parallel projections along 180 angular positions on a detector grid of size 128 , so that $q=180 \times 128$. To account for measurement uncertainty, the sinograms are degraded with a uniform noise with an amplitude $\chi$ equal to $2 \%$ of the maximal entry of $y$. We set manually the regularization parameter $\kappa$ so that it leads to a visually satisfactory decomposition: it is set to 0.25 for Glass and to 0.5 for Agaricus. The proposed algorithm PIPA-VM is compared to ADMM [72], which was the most competitive method in Section 4.1. Remark that, in order to make the implementation of ADMM feasible, we follow the same strategy as in [69], and alternate the minimization on the splitting variables. In our example, we need seven splitting variables. Since ADMM does not require a feasible starting point, we run it with two different initializations: ADMM1 refers to ADMM initialized like PIPA-VM, and ADMM2 refers to ADMM initialized with $x^{t}$ taken as the zero vector and $x^{g}$ set to $1 / 2\left(x_{\min }+x_{\max }\right) \mathbf{1}_{r}$.

\subsubsection{Results}

To compare the convergence speed of the different methods, we plot for each of them the relative distance between the current iterate $x$ and the final solution $x_{\infty}$, obtained after running the algorithms for 12 hours. As

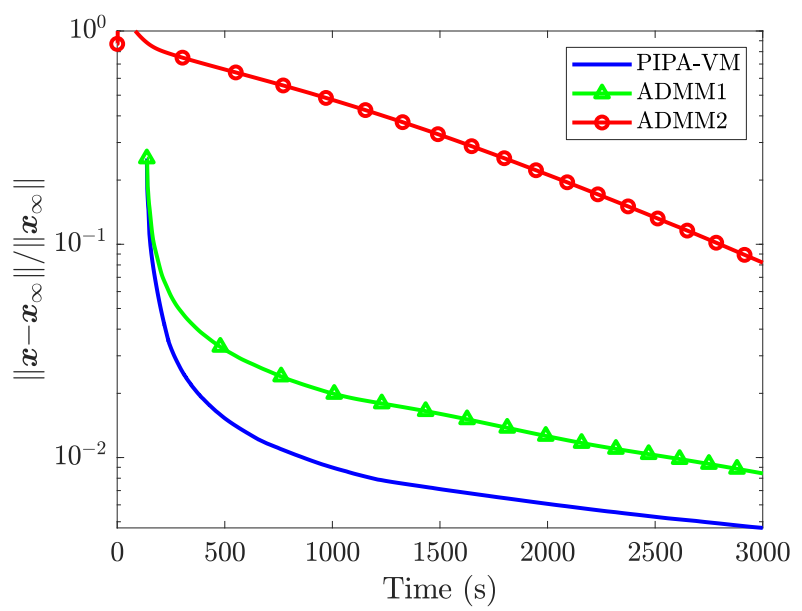

Fig. 6 Relative distance from the iterates to the limit point as a function of time for Glass.

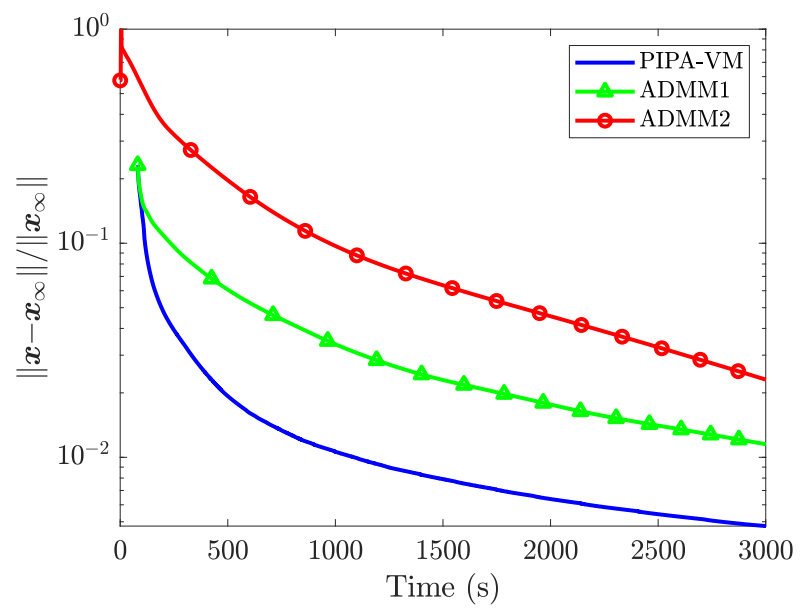

Fig. 7 Relative distance from the iterates to the limit point as a function of time for Agaricus.

one can see in Figures 6 and 7, PIPA-VM converges faster to its limit point than ADMM for both initializations. Remark that the time necessary to solve (56) and to find a feasible point is taken into account in the graphs. The results clearly show the advantage of using a feasible starting point over a simple initial guess.

To assess the visual quality of the geometry-texture decomposition we consider the solution obtained after reaching the stopping criterion $\left\|x-x_{\infty}\right\| /\left\|x_{\infty}\right\| \leq 10^{-2}$. This accuracy is reached first for PIPA-VM after $14 \mathrm{~min}$ for Glass and 18 min for Agaricus. The corresponding visual decomposition and reconstruction after these durations are given in Figure 8. As one can see in this figure, PIPA-VM identifies correctly the geometry as an almost piecewise-constant image, free from locally-fast varying components like the gills in the Agaricus mushroom. Moreover, the texture obtained for Glass image captures well the elements on the borders between the 

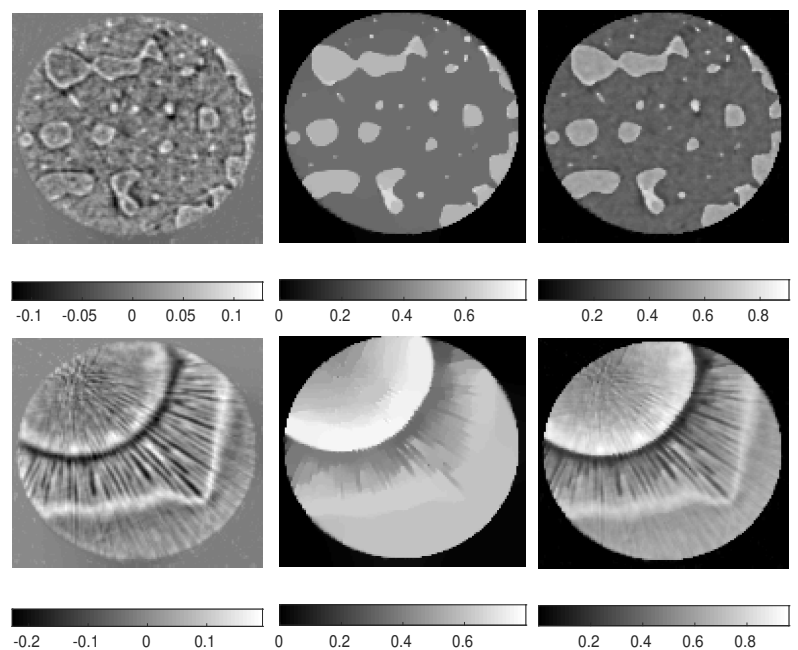

Fig. 8 Visual results for PIPA-VM. (Top) Glass obtained after 14 min. (Bottom) Agaricus obtained after 18 min. Left to right: texture, geometry, reconstruction $x^{t+g}$.

\begin{tabular}{rccc}
\hline & PIPA-VM & ADMM1 & ADMM2 \\
\hline Glass (14 min) & $\mathbf{1 9 . 0 0}$ & 18.74 & 18.69 \\
Agaricus (18 min) & $\mathbf{2 0 . 5 7}$ & 20.32 & 20.32 \\
\hline
\end{tabular}

Table 2 SNR $(\mathrm{dB})$ of the reconstruction $x^{t+g}$ obtained after running the algorithms for the same duration.

two species in presence, and the Agaricus gills can be found in the texture (Figure 8 bottom left).

Finally, we evaluate the reconstruction quality based on the signal-to-noise ratio:

$\mathrm{SNR}=20 \log _{10}\left(\left\|\bar{x}^{t+g}\right\| /\left\|x^{t+g}-\bar{x}^{t+g}\right\|\right)$,

where $\bar{x}^{t+g}$ denotes the ground-truth image. The SNR values obtained with the three methods for the reconstructions $x^{t+g}$ after the same durations are summarized in Table 2 .

It can be further observed in Figures 9 and 10, that even if the SNR converges to the same value for the three methods, PIPA-VM follows a path which would lead to a better SNR if stopped before convergence.

\section{Conclusion}

In this paper we have shown that it is possible to combine efficiently two powerful optimization frameworks: proximal splitting methods and interior point algorithms. One interesting feature of the resulting iterative method is the use of a variable metric, which can boost the convergence, as illustrated in our hyperspectral unmixing application. The convergence of the proposed method, as well as a convergence rate for the inner loop, have been obtained under suitable assumptions. As shown

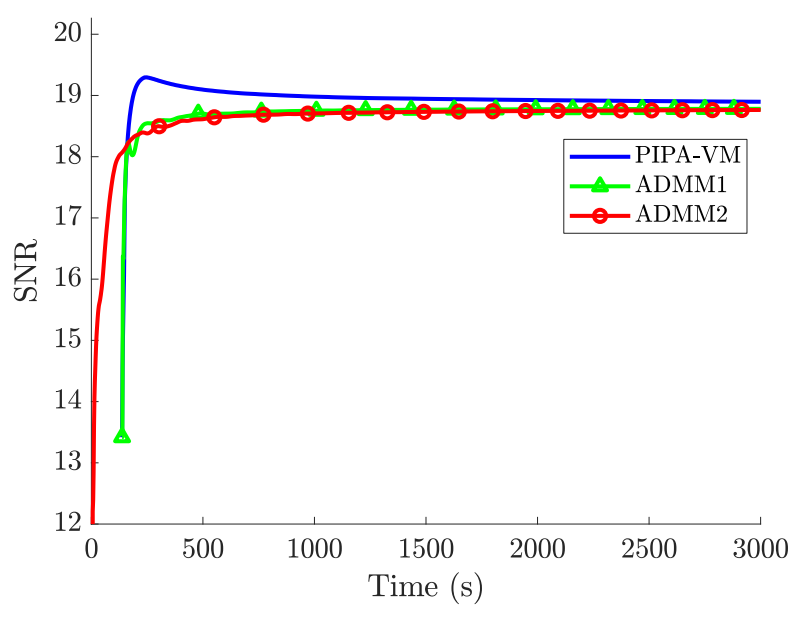

Fig. 9 SNR (dB) for $x^{t+g}$ as a function of time for Glass.

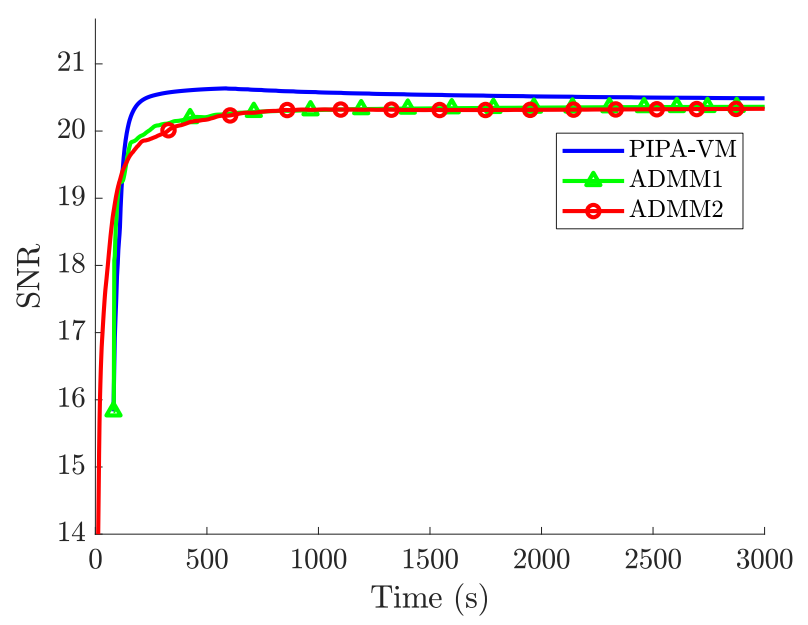

Fig. 10 SNR (dB) for $x^{t+g}$ as a function of time for Agaricus.

on two large-scale image processing applications, our method compares favorably in terms of speed of convergence with state-of-the-art algorithms. One future direction for further improvements would be to relax the convexity assumption in the mathematical analysis of PIPA, possibly by better relying on the KL property. This would widen the scope of application of this algorithm.

\section{References}

1. T. F. Chan and L. A. Vese. Active contours without edges. IEEE Transactions on Image Processing, 10(2): 266-277, 2001.

2. L. M. Briceño-Arias, G. Chierchia, E. Chouzenoux, and J.-C. Pesquet. A random block-coordinate DouglasRachford splitting method with low computational complexity for binary logistic regression. Computational $O p$ timization and Applications, pages 1-20, 2017. 
3. M. Nikolova. A variational approach to remove outliers and impulse noise. Journal of Mathematical Imaging and Vision, 20(1-2):99-120, 2004.

4. M.H. Wright. Interior methods for constrained optimization. Acta Numerica, pages 341-407, 1991.

5. A. Forsgren, P.E. Gill, and M.H. Wright. Interior methods for nonlinear optimization. SIAM review, 44(4):525$597,2002$.

6. J. Gondzio. Interior point methods 25 years later. European Journal of Operational Research, 218(3):587-601, 2012.

7. N. I. M. Gould, D. Orban, A. Sartenaer, and P. L. Toint. Superlinear convergence of primal-dual interior point algorithms for nonlinear programming. SIAM Journal on Optimization, 11(4):974-1002, 2001.

8. C. A. Johnson, J. Seidel, and A. Sofer. Interior-point methodology for 3-D PET reconstruction. IEEE Transactions on Medical Imaging, 19(4):271-285, 2000.

9. E. Chouzenoux, M. Legendre, S. Moussaoui, and J. Idier. Fast constrained least squares spectral unmixing using primal-dual interior-point optimization. IEEE Journal of Selected Topics in Applied Earth Observations and Remote Sensing, 7(1):59-69, 2014.

10. P. Armand, J.-C. Gilbert, and S. Jan-Jégou. A feasible BFGS interior point algorithm for solving convex minimization problems. SIAM Journal on Optimization, 11 (1):199-222, 2000.

11. S. Bonettini and T. Serafini. Non-negatively constrained image deblurring with an inexact interior point method. Journal of Computational and Applied Mathematics, 231 (1):236-248, 2009.

12. R. Ahmad and P. Schniter. Iteratively reweighted $\ell_{1}$ approaches to sparse composite regularization. IEEE Transactions on Computational Imaging, 1(4):220-235, 2015.

13. S. Osher, A. Sol, and L. Vese. Image decomposition and restoration using total variation minimization and the $H^{-1}$ norm. Multiscale Modeling \& Simulation, 1(3):349$370,2003$.

14. H. Fu, M.K. Ng, M. Nikolova, and J.L. Barlow. Efficient minimization methods of mixed $\ell 2-\ell 1$ and $\ell 1-\ell 1$ norms for image restoration. SIAM Journal on Scientific Computing, 27(6):1881-1902, 2006.

15. S.-J. Kim, K. Koh, M. Lustig, S. Boyd, and D. Gorinevsky. An interior-point method for large-scale $\ell_{1}$-regularized least squares. IEEE Journal of Selected Topics in Signal Processing, 1(4):606-617, 2007.

16. K. Fountoulakis and J. Gondzio. Performance of firstand second-order methods for $\ell_{1}$-regularized least squares problems. Computational Optimization and Applications, 65(3):605-635, 2016.

17. P.-L. Combettes and J.-C. Pesquet. Proximal splitting methods in signal processing. In Fixed-Point Algorithms for Inverse Problems in Science and Engineering, pages 185-212. Springer, 2011.

18. P.-L. Combettes and V.R. Wajs. Signal recovery by proximal forward-backward splitting. Multiscale Modeling $\&$ Simulation, 4(4):1168-1200, 2005.

19. G. H. G. Chen and R. T. Rockafellar. Convergence rates in forward-backward splitting. SIAM Journal on Optimization, 7(2):421-444, 1997.

20. P. L. Combettes and B. C. Vũ. Variable metric forwardbackward splitting with applications to monotone inclusions in duality. Optimization, 63(9):1289-1318, 2014.

21. E. Chouzenoux, J.-C. Pesquet, and A. Repetti. Variable metric forward-backward algorithm for minimizing the sum of a differentiable function and a convex function Journal of Optimization Theory and Applications, 162 (1):107-132, 2014.

22. P. Frankel, G. Garrigos, and J. Peypouquet. Splitting methods with variable metric for Kurdyka-Łojasiewicz functions and general convergence rates. Journal of Optimization Theory and Applications, 165(3):874-900, 2015.

23. S. Łojasiewicz. Une propriété topologique des sousensembles analytiques réels. Les équations aux dérivées partielles, 117:87-89, 1963.

24. K. Kurdyka. On gradients of functions definable in ominimal structures. In Annales de l'institut Fourier, volume 48, pages 769-783, 1998.

25. J. Bolte, A. Daniilidis, and A. Lewis. The Lojasiewicz inequality for nonsmooth subanalytic functions with applications to subgradient dynamical systems. SIAM Journal on Optimization, 17(4):1205-1223, 2007.

26. H. Attouch and J. Bolte. On the convergence of the proximal algorithm for nonsmooth functions involving analytic features. Mathematical Programming B, 116(1):516, 2009.

27. H. Attouch, J. Bolte, and B.F. Svaiter. Convergence of descent methods for semi-algebraic and tame problems: proximal algorithms, forward-backward splitting, and regularized Gauss-Seidel methods. Mathematical Programming, 137(1-2):91-129, 2013.

28. A. Kaplan and R. Tichatschke. Proximal methods in view of interior-point strategies. Journal of Optimization Theory and Applications, 98(2):399-429, 1998.

29. T. Valkonen. Interior-proximal primal-dual methods. arXiv preprint arXiv:1706.07067, 2017.

30. E. Chouzenoux, S. Moussaoui, and J. Idier. Majorizeminimize linesearch for inversion methods involving barrier function optimization. Inverse Problems, 28(6): 065011, 2012.

31. P. Tseng and S. Yun. A coordinate gradient descent method for nonsmooth separable minimization. Mathematical Programming, 117(1-2):387-423, 2009.

32. J.Y. Bello Cruz and T.T.A. Nghia. On the convergence of the forward-backward splitting method with linesearches. Optimization Methods and Software, 31(6): 1209-1238, 2016.

33. S. Bonettini, I. Loris, F. Porta, and M. Prato. Variable metric inexact line-search-based methods for nonsmooth optimization. SIAM journal on optimization, 26(2):891921, 2016.

34. S. Salzo. The variable metric forward-backward splitting algorithm under mild differentiability assumptions. SIAM Journal on Optimization, 27(4):2153-2181, 2017.

35. S. Bonettini and M. Prato. New convergence results for the scaled gradient projection method. Inverse Problems, 31(9), 2015.

36. R. T. Rockafellar and R. J.-B. Wets. Variational analysis, volume 317. Springer Science \& Business Media, 2009.

37. H. H. Bauschke and P. L. Combettes. Convex analysis and monotone operator theory in Hilbert spaces. Springer, 2017. doi: 10.1007/978-3-319-48311-5.

38. N. Pustelnik, C. Chaux, and J.-C. Pesquet. Parallel proximal algorithm for image restoration using hybrid regularization. IEEE Transactions on Image Processing, 20 (9):2450-2462, 2011.

39. J. D. Lee, B. Recht, N. Srebro, J. Tropp, and R. R. Salakhutdinov. Practical large-scale optimization for max-norm regularization. In 23rd Advances in Neural Information Processing Systems (NIPS), pages 1297-1305, Vancouver, Canada, Dec 2010. 
40. P.-L. Combettes, D. Dũng, and B.C. Vũ. Proximity for sums of composite functions. Journal of Mathematical Analysis and applications, 380(2):680-688, 2011.

41. F. Abboud, E. Chouzenoux, J.-C. Pesquet, J.-H. Chenot, and L. Laborelli. Dual block-coordinate forwardbackward algorithm with application to deconvolution and deinterlacing of video sequences. Journal of Mathematical Imaging and Vision, 59(3):415-431, 2017.

42. J. Bolte, S. Sabach, and M. Teboulle. Proximal alternating linearized minimization for nonconvex and nonsmooth problems. Mathematical Programming, 146(1-2): 459-494, 2014.

43. G. Li and T.K. Pong. Calculus of the exponent of Kurdyka--ojasiewicz inequality and its applications to linear convergence of first-order methods. Foundations of Computational Mathematics, 18(5):1199-1232, 2018.

44. H. Attouch, J. Bolte, P. Redont, and A. Soubeyran. Proximal alternating minimization and projection methods for nonconvex problems: An approach based on the Kurdyka-Łojasiewicz inequality. Mathematics of Operations Research, 35(2):438-457, 2010.

45. S. Harizanov, J.-C. Pesquet, and G. Steidl. Epigraphical projection for solving least squares Anscombe transformed constrained optimization problems. In 4 th International Conference on Scale Space and Variational Methods in Computer Vision (SSVM), pages 125-136, Schloss Seggau, Graz, Austria, Jun 2013. Springer.

46. O. Musse, F. Heitz, and J.-P. Armspach. Topology preserving deformable image matching using constrained hierarchical parametric models. IEEE Transactions on Image Processing, 10(7):1081-1093, 2001.

47. M. Klodt and D. Cremers. A convex framework for image segmentation with moment constraints. In 13th IEEE International Conference on Computer Vision (ICCV), pages 2236-2243, Sydney, Australia, Dec 2011.

48. E. Chouzenoux, J.-C. Pesquet, and A. Repetti. A block coordinate variable metric forward-backward algorithm. Journal of Global Optimization, 66(3):457-485, 2016.

49. H. Attouch, M.-O. Czarnecki, and J. Peypouquet. Proxpenalization and splitting methods for constrained variational problems. SIAM Journal on Optimization, 21(1): 149-173, 2011.

50. G. Garrigos, L. Rosasco, and S. Villa. Iterative regularization via dual diagonal descent. Journal of Mathematical Imaging and Vision, 60(2):189-215, 2018.

51. H. Attouch, A. Cabot, and M.-O. Czarnecki. Asymptotic behavior of nonautonomous monotone and subgradient evolution equations. Transactions of the American Mathematical Society, 370(2):755-790, 2018.

52. H. Attouch, M.-O. Czarnecki, and J. Peypouquet. Coupling forward-backward with penalty schemes and parallel splitting for constrained variational inequalities. SIAM Journal on Optimization, 21(4):1251-1274, 2011.

53. F. Alvarez and A. Cabot. Asymptotic selection of viscosity equilibria of semilinear evolution equations by the introduction of a slowly vanishing term. Discrete and Continuous Dynamical Systems, 15(3):921, 2006.

54. A. Cabot. Proximal point algorithm controlled by a slowly vanishing term: applications to hierarchical minimization. SIAM Journal on Optimization, 15(2):555572,2005

55. A.N. Iusem, B.F. Svaiter, and M. Teboulle. Entropy-like proximal methods in convex programming. Mathematics of Operations Research, 19(4):790-814, 1994.

56. A. S. Brito, J. X. da Cruz Neto, J. O. Lopes, and P. R. Oliveira. Interior proximal algorithm for quasiconvex pro- gramming problems and variational inequalities with linear constraints. Journal of Optimization Theory and Applications, 154(1):217-234, 2012.

57. E. A. P. Quiroz, L. M. Ramirez, and P. R. Oliveira. An inexact proximal method for quasiconvex minimization. European Journal of Operational Research, 246(3):721729, 2015.

58. N. Pustelnik, A. Benazza-Benhayia, Y. Zheng, and J.-C. Pesquet. Wavelet-based image deconvolution and reconstruction. Wiley Encyclopedia of Electrical and Electronics Engineering, pages 1-34, 1999.

59. C. Chaux, A. Benazza-Benyahia, J.-C. Pesquet, and L. Duval. Wavelet transform for the denoising of multivariate images. In C. Collet, J. Chanussot, and K. Chehdi, editors, Multivariate Image Processing, pages 203-237. ISTE Ltd and John Wiley \& Sons Inc, 2010.

60. L.I. Rudin, S. Osher, and E. Fatemi. Nonlinear total variation based noise removal algorithms. Physica D: Nonlinear Phenomena, 60(1-4):259-268, 1992.

61. T. Hastie, R. Tibshirani, and J. H. Friedman. The elements of statistical learning: data mining, inference, and prediction. Springer, 2009.

62. S. Boyd and L. Vandenberghe. Convex optimization. Cambridge University Press, 2004.

63. Y. Huang and Y. Dong. New properties of forwardbackward splitting and a practical proximal-descent algorithm. Applied Mathematics and Computation, 237: 60-68, 2014

64. J.-F. Bonnans, J.-C. Gilbert, C. Lemaréchal, and C.A. Sagastizábal. Numerical optimization: theoretical and practical aspects. Springer Science \& Business Media, 2006.

65. M.-C. Corbineau, E. Chouzenoux, and J.-C. Pesquet. PIPA: a new proximal interior point algorithm for largescale convex optimization. In 43rd IEEE International Conference on Acoustics, Speech and Signal Processing (ICASSP), pages 1343-1347, Calgary, Canada, Apr 2018.

66. M.-C. Corbineau, E. Chouzenoux, and J.-C. Pesquet. Geometry-texture decomposition/reconstruction using a proximal interior point algorithm. In 10th IEEE Sensor Array and Multichannel Signal Processing Workshop (SAM), pages 435-439, Sheffield, UK, Jul 2018.

67. J. M. Bioucas-Dias, A. Plaza, N. Dobigeon, M. Parente, Q. Du, P. Gader, and J. Chanussot. Hyperspectral unmixing overview: Geometrical, statistical, and sparse regression-based approaches. IEEE Journal of Selected Topics in Applied Earth Observations and Remote Sensing, 5(2):354-379, 2012.

68. R. H. Chan, K. K. Kan, M. Nikolova, and R. J. Plemmons. A two-stage method for spectral-spatial classification of hyperspectral images. arXiv preprint arXiv:1806.00836, 2018.

69. M.-D. Iordache, J.M. Bioucas-Dias, and A. Plaza. Total variation spatial regularization for sparse hyperspectral unmixing. IEEE Transactions on Geoscience and Remote Sensing, 50(11):4484-4502, 2012.

70. N. Keshava and J. F. Mustard. Spectral unmixing. IEEE Signal Processing Magazine, 19(1):44-57, 2002.

71. S. Becker and J. Fadili. A quasi-Newton proximal splitting method. In 25th Advances in Neural Information Processing Systems (NIPS), pages 2618-2626, Lake Tahoe, USA, Dec 2012.

72. S. Setzer, G. Steidl, and T. Teuber. Deblurring Poissonian images by split Bregman techniques. Journal of Visual Communication and Image Representation, 21(3): 193-199, 2010. 
73. N. Komodakis and J.-C. Pesquet. Playing with duality: An overview of recent primal-dual approaches for solving large-scale optimization problems. IEEE Signal Processing Magazine, 32(6):31-54, 2015.

74. P. L. Combettes, L. Condat, J.-C. Pesquet, and B. C. Vũ. A forward-backward view of some primal-dual optimization methods in image recovery. In 21st IEEE International Conference on Image Processing (ICIP), pages 4141-4145, Paris, France, Oct 2014.

75. H. Raguet, J. Fadili, and G. Peyré. A generalized forward-backward splitting. SIAM Journal on Imaging Sciences, 6(3):1199-1226, 2013.

76. R. Shefi and M. Teboulle. Rate of convergence analysis of decomposition methods based on the proximal method of multipliers for convex minimization. SIAM Journal on Optimization, 24(1):269-297, 2014.

77. H. Raguet and L. Landrieu. Preconditioning of a generalized forward-backward splitting and application to optimization on graphs. SIAM Journal on Imaging Sciences, 8(4):2706-2739, 2015.

78. J. Frecon, N. Pustelnik, H. Wendt, L. Condat, and P. Abry. Multifractal-based texture segmentation using variational procedure. In 12th IEEE Image, Video, and Multidimensional Signal Processing Workshop (IVMSP), pages 1-5, 2016.

79. J.-F. Aujol and T. F. Chan. Combining geometrical and textured information to perform image classification. Journal of Visual Communication and Image Representation, 17(5):1004-1023, 2006.

80. M. Bertalmio, L. Vese, G. Sapiro, and S. Osher. Simultaneous structure and texture image inpainting. IEEE Transactions on Image Processing, 12(8):882-889, 2003.

81. L. M. Briceño-Arias, P. L. Combettes, J.-C. Pesquet, and N. Pustelnik. Proximal algorithms for multicomponent image recovery problems. Journal of Mathematical Imaging and Vision, 41(1-2):3-22, 2011.

82. N. Pustelnik, H. Wendt, and P. Abry. Local regularity for texture segmentation: Combining wavelet leaders and proximal minimization. In 38th IEEE International Conference on Acoustics, Speech and Signal Processing (ICASSP), pages 5348-5352, Vancouver, Canada, May 2013.

83. R. M. Haralick. Statistical and structural approaches to texture. Proceedings of the IEEE, 67(5):768-804, 1979.

84. A. C. Kak and M. Stanley. Principles of Computerized Tomographic Imaging. SIAM, 2001.

85. E. Chouzenoux, F. Zolyniak, E. Gouillart, and H. Talbot. A majorize-minimize memory gradient algorithm applied to X-ray tomography. In 20th IEEE International Conference on Image Processing (ICIP), pages 1011-1015, Melbourne, Australia, Sep 2013.

86. E. Gouillart, F. Krzakala, M. Mézard, and L. Zdeborová. Belief-propagation reconstruction for discrete tomography. Inverse Problems, 29(3):035003, 2013.

87. E. Chouzenoux, J.-C. Pesquet, and A. Repetti. Variable metric forwardbackward algorithm for minimizing the sum of a differentiable function and a convex function. Journal of Optimization Theory and Applications, 162(1):107-132, 2014.

88. A. Chambolle and T. Pock. A first-order primal-dual algorithm for convex problems with applications to imaging. Journal of Mathematical Imaging and Vision, 40(1): 120-145, 2011. 